\title{
Liquid crystalline assembly for potential combinatorial chemo-herbal drug delivery to lung cancer cells
}

This article was published in the following Dove Medical Press journal: International Journal of Nanomedicine

Hadeer M Abdelaziz, ${ }^{1,2}$ Ahmed O Elzoghby, ${ }^{1,3-5}$ Maged W Helmy, ${ }^{1,6}$ Magda W Samaha, 1,3 Jia-You Fang, ${ }^{7-9}$ May S Freag ${ }^{1,4,5,10}$

'Cancer Nanotechnology Research Laboratory (CNRL), Faculty of Pharmacy, Alexandria University, Alexandria 21521, Egypt; ${ }^{2}$ Department of Pharmaceutics, Faculty of Pharmacy, Damanhur University, Pharmacy, Faculty of Pharmacy, Alexandria University, Alexandria 21521, Egypt; ${ }^{4}$ Division of Engineering in Medicine, Department of Medicine, Brigham and Women's Hospital, Harvard Medical School, Boston, MA 02 II5, USA; ${ }^{5}$ HarvardTechnology (HST), Cambridge, MA 02I39, USA; ${ }^{6}$ Department of Pharmacology and Toxicology, Faculty of Pharmacy, Damanhur University, Damanhur, Egypt; 'Pharmaceutics Laboratory, Graduate Institute of Natural Products, Chang Gung University, Taoyuan 333, Taiwan; ${ }^{8}$ Research Center for Industry of Human Ecology and Research Center for Chinese Herbal Medicine, Chang Gung University of Science and Technology, Kweishan, Taoyuan 333, Taiwan; ${ }^{9}$ Department of Anesthesiology, Chang Gung Memorial Hospital, Kweishan, Taoyuan 333, Taiwan; ${ }^{10}$ Department of Pharmaceutics, Faculty of Pharmacy, Alexandria University, Alexandria 21521, Egypt

Correspondence: May S Freag

Department of Pharmaceutics, Faculty of Pharmacy, Alexandria University, I-azarita square, Alexandria 2152I, Egypt

$\mathrm{Tel}+20106503472$ ।

$\mathrm{Fax}+2034873273$

Email may.s.freag@alexu.edu.eg

Jia-You Fang

Pharmaceutics Laboratory, Graduate Institute of Natural Products, Chang Gung University,

Taoyuan 333, Taiwan Damanhur, Egypt; ${ }^{3}$ Department of Industrial MIT Division of Health Sciences and

Email fajy@mail.cgu.edu.tw

Background: Lung cancer is the most common cancer and the leading cause of total deaths worldwide. Its classified into two major types including non-small cell lung carcinoma (NSCLC) and small cell lung carcinoma (SCLC) based on the origin of abnormal lung cells as well as the smoking status of the patient. NSCLC is the most common and aggressive type of lung cancer representing $80 \%-85 \%$ of all cases.

Purpose: The aim of the study was to present lyotropic liquid crystalline nanoparticles (LCNPs) as promising carriers for co-delivery of the chemotherapeutic agent, pemetrexed (PMX) and the herbal drug, resveratrol (RSV) for effective lung cancer management.

Methods: The proposed PMX-RSV-LCNPs were prepared by hydrotrope method. Hydrophobic ion pairing with cetyl trimethyl ammonium bromide (CTAB) was implemented to increase the encapsulation efficiency of the hydrophilic PMX up to $95 \% \pm 3.01 \%$.

Results: The tailored PMX-RSV-LCNPs exhibited a particle size of $173 \pm 0.26 \mathrm{~nm}$ and biphasic release pattern with a relatively initial burst release within first 3-4 hour followed by sustained release up to 24 hours. Moreover, PMX-RSV-LCNPs manifested superior concentration and time dependent cytotoxicity profile against A549 lung cancer cells with $\mathrm{IC}_{50} 4.0628 \mu \mathrm{g} / \mathrm{mL}$. Besides, the enhanced cellular uptake profile based on bioadhesive properties of glyceryl monoolein (GMO) as well as energy independent (cholesterol dependent) pattern. In-vivo evaluations against urethane induced lung cancer bearing mice demonstrated the potentiality of PMX-RSV-LCNPs in tumor growth inhibition via inhibition of angiogenesis and induction of apoptosis. The results were supported by histopathological analysis and immunohistochemical Ki67 staining. Moreover, PMX-RSV-LCNPs displayed a promising safety profile via attenuating nephro- and hepatotoxicity. Conclusion: PMX-RSV-LCNPs elaborated in the current study hold a great promise for lung cancer treatment.

Keywords: hydrophobic ion pairing, liquid crystalline nanoparticles, lung cancer, glyceryl monoolein, pemetrexed, resveratrol

\section{Introduction}

Lung cancer is one of the most dreadful cancers for both men and women. ${ }^{1}$ American Cancer Society estimated that in 2018 lung and bronchus cancers would be responsible for 234,030 new cases which represent $14 \%$ of all new cancer cases and 154,050 deaths. ${ }^{2}$ Lung cancer is mainly classified into non-small-cell lung cancer (NSCLC; major and common form responsible for $85 \%$ cases) and small-cell lung carcinoma., Despite the researcher's attempts to improve outcomes among lung cancer patients, results are still unsatisfactory. ${ }^{5}$ For advanced stages of lung cancer, chemotherapy is considered the first line of treatment which is usually administered intravenously via systemic circulation. ${ }^{4,6}$ However, conventional chemotherapy either used alone or in combination with others 
faces many obstacles that limit its use. Most of the chemotherapeutic agents have poor aqueous solubility, lack of selectivity, and extensive metabolism via first-pass effect which in turn require higher doses to achieve the therapeutic level that subsequently affect tumor cells as well as normal healthy cells. ${ }^{7-9}$

Alimta $^{\circledR}$ (pemetrexed, PMX, Eli Lilly and Company, Indianapolis, Indiana, USA), a promising Food and Drug Administration (FDA)-approved chemotherapeutic agent as monotherapy for the maintenance treatment of NSCLC, is also considered as the first-line treatment in combination with cisplatin. ${ }^{10}$ PMX disodium $\left(\mathrm{C}_{20} \mathrm{H}_{19} \mathrm{~N}_{5} \mathrm{Na}_{2} \mathrm{O}_{6}\right)$ is a white freely water-soluble crystalline powder with a molecular weight of $471.37 \mathrm{~g} / \mathrm{mol} .{ }^{11} \mathrm{PMX}$ is a potent multi-targeted antifolate agent that exerts its action by disrupting de novo biosynthesis of thymidine and purine nucleotides essential for cell replication. The use of PMX, either as monotherapy or in combination, is accompanied with many undesirable adverse effects including bone marrow suppression manifested as anemia, neutropenia, leukopenia, and thrombocytopenia as well as nephro- and hepatotoxicities. ${ }^{12-16}$ Moreover, PMX is rapidly eliminated and unchanged by renal tubular secretion and to a lesser extent by glomerular filtration. In addition, it undergoes limited hepatic metabolism with terminal half-life between 2 and 5 hours. ${ }^{12}$ Therefore, encapsulating PMX in a suitable drug delivery system seems to be a promising approach to surmount PMX pharmaceutical obstacles and improve its clinical utility.

Recently, the use of herbal drugs has magnetized the researchers' attention based on their great tendency in sensitizing tumor cells and imparting synergistic activity to chemotherapeutic agents. ${ }^{17-19}$ Among promising herbal drugs, resveratrol (RSV) proved its great capability in protecting against cancer. ${ }^{20} \mathrm{RSV}\left(3,5,4^{\prime}\right.$-trihydroxystilbene) is a polyphenolic phytoalexin produced in various plants in response to stress. ${ }^{21,22}$ RSV has several activities including antioxidant, anti-inflammatory, proapoptotic, antiproliferative, antiangiogenesis, and chemopreventive properties. ${ }^{21-24}$ In addition, it plays a vital role in overcoming multidrug resistance (MDR) associated with most of the chemotherapeutic agents. This was attributed to its ability to prohibit P-glycoprotein (P-gp) activity, thus potentiating intracellular accumulation of coadministered chemotherapeutic payloads. ${ }^{25,26} \mathrm{~A}$ promising synergistic combination of RSV and PMX was previously reported by Chen et al, ${ }^{27}$ where PMX significantly decreased the expression of ERCC1, phospho-p38 MAPK protein levels, and the DNA repair capacity, thus enhancing the RSV-induced cytotoxic effect on NSCLC cells and proposing the potential outcomes of coadministering PMX and RSV for the treatment of lung carcinoma. However, RSV has many limitations including instability, poor water solubility, low bioavailability, and short biological half-time. In addition, rapid and extensive metabolism hindered the anticancer activity of RSV even in high doses. ${ }^{28}$ Therefore, optimizing a nanoscale drug delivery system encapsulating both drugs could be of prime importance for enhanced lung cancer treatment.

Recently, researchers pay great attention to lyotropic liquid crystalline nanoparticles (LCNPs) as new lipid-based drug delivery systems for cancer therapy. They are self-assembled structures formed when several polar lipids including Glyceryl monooleate (GMO), phytantriol, glycolipids, and glycerates were dispersed in water in the presence of stabilizers. LCNPs possess bicontinuous lipid bilayer enclosing water channels. Their unique multi-compartmental structure enables them to efficiently deliver hydrophilic, hydrophobic, or amphiphilic molecules. ${ }^{29-31}$ The biocompatibility, biodegradability, bioadhesion, and prolonged drug release characteristics make LCNPs suitable carriers for cancer therapy. Their inherent small particle size (PS) facilitates passive targeting via enhanced permeation and retention effect. ${ }^{32-35}$ Recently, Freag et $\mathrm{al}^{30}$ successfully formulated surface-modified PEGylated aloe-emodin-loaded LCNPs (AE-polyethylene glycol [PEG]LCNPs) for breast cancer treatment. The LCNPs investigated in their study demonstrated improved hemocompatibility, increased serum stability, and enhanced cytotoxicity and cellular uptake in breast cancer cells compared to free drug.

In view of the abovementioned information, the current study focused, for the first time, on the fabrication of LCNPs for the combined delivery of both PMX and RSV. The proposed formulation was evaluated in vitro and in vivo for their efficacy in lung cancer treatment. In vitro cytotoxicity and cellular uptake studies were performed using A549 lung carcinoma cells. In addition, in vivo antitumor activity of PMX-RSV-LCNPs was evaluated in urethane-induced lung cancer-bearing mice.

\section{Materials and methods Materials}

PMX (purity 98\%) and RSV were purchased from Baoji Guokang Bio-Technology Co., Ltd (Baoji, People's Republic of China). Peceol ${ }^{\circledR}$ (GMO) was kindly provided by Gattefosse (Saint-Priest, France). Human A549 lung cancer cell line was purchased from Egyptian Organization for Biological Products and Vaccines (VACSERA Holding Company, Giza Governorate, Egypt). Poloxamer-407 (P407), cetyltrimethylammonium bromide (CTAB), DMEM, FBS, and MTT were purchased from Sigma-Aldrich Co. (St Louis, MO, USA). Caspase 3 (CASP-3) ELISA Kit was purchased from WKEA Med 
Supplies Corp. (Chaoyang District, Changchun, Jilin, People's Republic of China). Vascular endothelial growth factor (VEGF) ELISA kit, RayBio ${ }^{\circledR}$ (catalog number: ELR-VEGF-001) was purchased from RayBio Tech Inc. (Norcross, GA, USA). All other reagents and chemicals were of analytical grade.

\section{Methodology}

Preparation of PMX-RSV-loaded LCNPs

PMX-RSV-LCNPs were fabricated via the hydrotrope method. ${ }^{36}$ Briefly, RSV $(10 \mathrm{mg})$ was dissolved in an isotropic mixture of $200 \mathrm{mg}$ GMO and $0.18 \mathrm{~mL}$ absolute ethanol. The resulting isotropic solution was added dropwise onto $2 \mathrm{~mL}$ of P407 solution $(0.5 \% \mathrm{w} / \mathrm{v})$ containing $10 \mathrm{mg}$ PMX under magnetic stirring. The mixture was equilibrated at room temperature for 24 hours and then dispersed into another $6 \mathrm{~mL}$ of P407 solution $(0.5 \% \mathrm{w} / \mathrm{v})$ followed by homogenization at 13,000 rpm for 3 minutes to finally obtain the LCNP dispersion.

Different formulation variables including the type of hydrotrope (ethanol, PEG 400, propylene glycol), concentration of ethanol, P407 concentration, and percentage loading of PMX and RSV were investigated. The influence of different variables on PS, size distribution, zeta potential, and encapsulation efficiency of the prepared LCNPs was investigated.

For cellular uptake studies, fluorescent-labeled LCNPs were prepared, where $0.08 \% \mathrm{w} / \mathrm{w}$ coumarin- 6 (with respect to lipid) was added to the isotropic mixture of GMO and absolute ethanol and then processed as mentioned earlier.

\section{Preparation of ion-paired PMX-RSV- loaded LCNPs}

To increase the hydrophobicity of PMX and improve its lipid solubility, in situ hydrophobic ion pairing (HIP) of PMX with the cationic surfactant CTAB was performed following the previously reported protocol. ${ }^{37,38}$ Briefly, $34 \mathrm{mg}$ of CTAB was added with $10 \mathrm{mg}$ PMX into P407 solution prior to the addition of the isotropic (GMO and ethanol) mixture and then processed as mentioned earlier. Optimization of the in situ ion-pairing process involved the investigation of different PMX:CTAB molar ratios of 1:2 and 1:4 on the $\%$ entrapment efficiency (\% EE) of PMX.

\section{Development of novel HPLC method for simultaneous determination of PMX and RSV}

A novel, accurate, precise, and sensitive HPLC method was developed and validated for simultaneous determination of both RSV and PMX using Agilent 1260 Infinity HPLC system. Details of the test are provided in Supplementary materials.

\section{Characterization of LCNPs EE of PMX and RSV}

The \%EE of different LCNP formulations was determined using the dialysis bag method following a protocol previously reported by Swarnakar et a ${ }^{39}$ Briefly, $1 \mathrm{~mL}$ of LCNP dispersion was transferred to dialysis bag. The bags were then placed in $50 \mathrm{~mL}$ PBS and subjected to centrifugation (Model 3 K-30; Sigma Laboratory Refrigerated Centrifuge, Osterode am Harz, Germany) at $500 \mathrm{rpm}$ for 30 minutes to separate free PMX and RSV. The procedure was optimized and validated in-house for complete separation of free drugs from the formulation. The actual concentrations of PMX and RSV entrapped within LCNPs represent the difference between the initial amounts added during LCNP fabrication and free unentrapped drug separated after centrifugation (Equation 1). The concentrations of PMX and RSV were quantified using HPLC at $\lambda_{\text {max }}=225$ and $306 \mathrm{~nm}$, respectively.

$$
\% \mathrm{EE}=\frac{\text { Amount of drug entrapped in LCNPs }}{\text { Initial amount of drug }} \times 100
$$

\section{PS and zeta potential}

The mean PS, polydispersity index (PDI), and zeta potential ( $\delta$-potential) of LCNP formulations were measured via dynamic light scattering (DLS) and electrophoretic mobility technique using Zetasizer (Nano ZS/ZEN3600 Zetasizer; Malvern Instruments, Malvern, UK). The samples were diluted with distilled water (1:50) to obtain final experimental values in triplicate at room temperature. Results were shown as mean size \pm SD. ${ }^{40}$

\section{Study of the phase behavior of LCNPs}

Investigation of the phase behavior of both placebo and dual drug-loaded formulations was performed using a polarizing microscope. Micron-sized liquid crystalline particles were fabricated as described earlier but without the size reduction homogenization step. After that, a drop of the dispersion was placed onto a slide and imaged under polarizing light microscope (Zeiss Axiovert 40 MAT microscope fitted with camera) at magnification power of $100 \times$ to evaluate the occurrence of birefringence. ${ }^{41}$

\section{Transmission electron microscopy (TEM)}

The morphology of ion-paired PMX-RSV-LCNPs was examined via TEM (Jeol JEM-2100; JEOL, Tokyo, Japan) at an accelerating voltage of $80 \mathrm{kV} .{ }^{42}$ After sample dilution with distilled water (1:20), a drop of the sample was placed onto 
a carbon-coated copper grid and left for air-drying. Then, the films were visualized under TEM and photographed.

\section{In vitro release study}

In vitro drug release profile from different LCNP formulations was performed using the dialysis bag method..$^{43}$ Samples investigated encompassed free aqueous solution of PMX, free ethanolic solution of RSV, PMX-RSV-LCNPs (F8), and ion-paired PXM-RSV-LCNPs (F10). Samples were added into a sealed dialysis bag (molecular weight cutoff [MWCO]: 12-14 kDa). The dialysis bags were placed in $100 \mathrm{~mL}$ phosphate buffer, $\mathrm{pH}$ 7.4 , and incubated at $37^{\circ} \mathrm{C}$ with a shaking speed of $100 \mathrm{rpm}$ in shaking water bath. Aliquots $(2 \mathrm{~mL})$ were withdrawn at different time intervals $(0.25,0.5,1,2,4,6,8$, and 24 hours $)$ followed by compensation with the same volume of fresh release medium. The samples were filtered using $0.45 \mu \mathrm{m}$ Millipore filter (Millipore ${ }^{\circledR}$; Thermo Fisher Scientific, Waltham, MA, USA) and then quantified for their PMX and RSV content using validated HPLC method as mentioned earlier.

\section{In vitro anticancer study}

\section{Cell culture}

A lung carcinoma cell line A549 was used for examining in vitro cytotoxicity of the proposed formulations. For the propagation of A549 cells, American Type Culture Collection (ATCC) complete growth medium (Ham's F12K medium with $2 \mathrm{mM}$ L-glutamine adjusted to contain $1.5 \mathrm{~g} / \mathrm{L}$ sodium bicarbonate, 90\%; FBS, 10\%) was utilized. The cells were grown in $75 \mathrm{~mL}$ flasks in an atmosphere of $5 \% \mathrm{CO}_{2}$ and $100 \%$ relative humidity and subcultured two to three times per week.

\section{In vitro cytotoxicity}

In vitro cytotoxicity of free PMX, free RSV, free combined PMX/RSV solution, blank LCNPs, and ion-paired PMXRSV-LCNPs (F10) was evaluated in A549 lung cancer cells using MTT assay. ${ }^{44}$ Details of the test are provided in Supplementary materials.

\section{In vitro cellular uptake}

A549 lung cancer cells were seeded on CELL view ${ }^{\text {TM }}$ slide at a density of 3,000 cells/well and left overnight to allow enough time for cell attachment. Cells were incubated with fresh medium containing free coumarin- 6 dye and coumarin6 -loaded LCNPs at the concentration of $0.08 \% \mathrm{w} / \mathrm{w}$ coumarin-6 (with respect to lipid). Details of the test are provided in Supplementary materials.

\section{In vivo study}

\section{Animals}

The guidelines developed by the Animal Care and Use Committee, Faculty of Pharmacy, Alexandria University, Alexandria, Egypt, and in accordance with regulations of the National Research Council's guide for the care and use of laboratory animals were adopted for in vivo study. Twentyeight Balb/c male mice (15 $\pm 5 \mathrm{~g}, 3-4$ weeks) were housed in stainless steel cages in four groups (seven mice per group). The animals were kept under standard environmental conditions $\left(23^{\circ} \mathrm{C} \pm 1^{\circ} \mathrm{C}, 55 \% \pm 5 \%\right.$ humidity and 12 hours $/ 12$ hours light/dark cycle) and maintained with free access to standard laboratory diet (balanced nutrient of chow and water). Lung cancer was induced within mice via intraperitoneal injection of chemical carcinogen and urethane (ethyl carbamate, $1 \mathrm{~g} / \mathrm{kg})$ followed by post-dose of urethane $(0.5 \mathrm{~g} / \mathrm{kg})$ after 15 days. The mice were left for 12-16 weeks to ensure complete induction of lung cancer. After that, experimental model of mice was sacrificed to evaluate tumor incidence and precancerous lesions prior to exposing rest of mice to treatment. The excised lungs were stained with H\&E and undergone histopathological and immunohistochemical investigations.

\section{Evaluation of antitumor efficacy}

After tumor induction, 28 mice were classified randomly into four groups, 7 mice each. The groups encompassed as follows: positive control group (untreated tumor-bearing mice), negative control group (healthy mice treated with saline), free PMX/RSV-treated group, and ion-paired PMX/ RSV-LCNP (F10)-treated group. All animals were administered their formulation via intravenous (IV) route into the tail vein. Animals of the last two groups were injected with equivalent concentration of PMX and RSV $(0.94 \mathrm{mg} / \mathrm{kg})$ twice per week for 21 days. Free PMX/RSV mixture was dissolved in cosolvent (dimethyl sulfoxide/PEG 400/saline $0.5 / 4.5 / 5 \mathrm{v} / \mathrm{v} / \mathrm{v})$. At 25 th day, the mice were sacrificed and the excised lungs were weighted and divided for tumor growth biomarker measurement as well as histopathological and immunohistochemical examinations.

\section{Evaluation of tumor growth biomarkers}

The excised parts of lung tumors were carefully weighed and homogenized in cold PBS using tissue homogenizer to make a final $40 \%$ tissue homogenate. This homogenate was centrifuged at $1,700 \mathrm{rpm}$ at $4^{\circ} \mathrm{C}$ for 10 minutes, and the supernatants were divided into separate small aliquots and stored at $-80^{\circ} \mathrm{C}$ for further quantitative determination of 
tumor growth biomarkers. The degree of angiogenesis was determined via detecting the level of the angiogenic VEGF using "VEGF ELISA Kit" (RayBio Tech Inc.), while the induction of apoptosis was confirmed by determining the level of tissue CASP-3 using "caspase-3 (CASP-3) ELISA Kit" (WKEA Med Supplies Co.). All the markers were quantified according to the manufacturer's protocol.

\section{Histopathological studies}

A part of excised lung tumors was preserved and fixed with $10 \%$ neutral formalin for 24 hours, then stained with hematoxylin for 5 minutes and eosin for 2 minutes (H\&E), dehydrated in alcohol, and mounted in Canada balsam before microscopical examination. The tumors obtained from different groups were examined in ten random sections $(\times 40)$ for histopathological neoplastic changes. Moreover, the average number of microscopic metastatic lung foci and their diameter were determined.

\section{Immunohistochemical analysis}

The previously conserved excised lungs in formalin and embedded in paraffin as blocks were cut and rinsed using xylene and then rehydrated before staining steps. Methanolic solution of hydrogen peroxide $(7 \%)$ was added for 20 minutes, then left for 5 minutes in heated citrate phosphate buffer ( $\mathrm{pH}$ 6.0) using microwave then immersed in milk/ distilled water $(5 \%)$ for 45 minutes. Individual treatment of sections was performed using Ki-67 antibody at $4{ }^{\circ} \mathrm{C}$ overnight. After that, the sections were washed and retreated with a secondary anti-mouse biotinylated antibody in a dark room for 1 hour. Nuclear staining was carried out to localize cellular Ki-67 immunoreactivity. Each tumor sample was examined for Ki-67 expressions in ten random sections $(\times 40)$ in which the number of positive cells/total was calculated. Moderate-to-strong brownish cellular cytoplasmic staining was accounted for positive, whereas unstained or faint stained cellular cytoplasm was accounted for negative.

\section{Statistical analyses}

Statistical analysis of in vitro results was carried out using Student's $t$-test ( $P \leq 0.05$; GraphPad Prism version 3.02; GraphPad Software, Inc., La Jolla, CA, USA). However, the results of the in vivo antitumor activity were analyzed using IBM SPSS Software package (version 20; IBM Corporation, Armonk, NY, USA). Statistical significance between the groups was analyzed using ANOVA and Tukey's multiple comparison tests. Statistical significance was judged at the $5 \%$ level.

\section{Results and discussion Preparation of ion-paired PMX-RSV-LCNPs}

The aim of the current study was to develop lyotropic LCNPs for the co-delivery of PMX and RSV. Thanks to the unique structure of LCNPs which facilitate incorporation of different types of drugs, both hydrophilic (PMX) and lipophilic (RSV) drugs were successfully loaded in the LCNPs. Herein, the hydrotrope method, previously developed by Spicer et al, ${ }^{36}$ was adopted for the fabrication of LCNPs in which a hydrotrope was used to increase the aqueous solubility of poorly soluble GMO lipid. The dropwise addition of this hydrotropic mixture to water containing P407 resulted in gradual decrease in the solubility of GMO resulting in instantaneous formation of stable liquid crystalline particles in a nanometric range. In addition, the hydrotrope method guaranteed the stability of the thermosensitive drug RSV bypassing the drastic conditions accompanied by conventional method including elevated temperature and pressure. The composition of the prepared LCNP formulations (F1-F10) is summarized in Table 1. As given in the table, PMX (F8) demonstrated relatively low $\% \mathrm{EE}$ due to its anionic nature and high aqueous solubility (log $P 1.54)$ that could result in its poor entrapment into lipid-based systems and subsequent partitioning into the continuous aqueous phase. Generally, sustained release of water-soluble drugs from nanocarrier delivery systems remains a challenging task. Many attempts have been exploited to overcome this challenge including chemical cross-linking and HIP. ${ }^{45,46}$ Therefore, in the current study, the HIP technique was applied to enhance the hydrophobicity of PMX and enhance its EE. ${ }^{38}$ HIP technique is simply performed by the interaction between ionic drug and the opposite ionic head group of a fatty acid, surface-active agents, or other amphiphilic molecules at suitable $\mathrm{pH}$ without the modification of their chemical structures. In the current study, CTAB was selected as a counter-cationic surfaceactive agent for in situ formation of HIP with PMX. CTAB was added to the aqueous phase containing PMX, and the influence of different PMX:CTAB molar ratios on the $\% \mathrm{EE}$ was investigated. As summarized in Table 1, 1:4 PMX:CTAB molar ratio (F10) showed the highest \%EE of PMX (95\%), so it was selected as an optimum ratio for further studies.

\section{Physicochemical characterization of LCNPs \\ PS \\ Effect of different hydrotropes}

The effect of different hydrotropes including propylene glycol, PEG 400, and absolute ethanol on the quality attributes 
Table I Composition and physicochemical characterization of placebo and PMX-RSV-loaded LCNPs

\begin{tabular}{|c|c|c|c|c|c|c|c|c|c|}
\hline Formula & $\begin{array}{l}\text { Hydrotrope } \\
\text { (\% w/v) }\end{array}$ & $\begin{array}{l}\text { P407a } \\
\text { (\% w/v) }\end{array}$ & $\begin{array}{l}\text { PMX } \\
\text { (mg) }\end{array}$ & $\begin{array}{l}\text { RSV } \\
\text { (mg) }\end{array}$ & $\begin{array}{l}\text { Size } \\
(\mathrm{nm})\end{array}$ & PDI & $\begin{array}{l}\text { Zeta potential } \\
(\mathrm{mV})\end{array}$ & $\begin{array}{l}\text { RSV } \\
\text { \%EE }\end{array}$ & $\begin{array}{l}\text { PMX } \\
\text { \%EE }\end{array}$ \\
\hline$\overline{\mathrm{FI}^{\mathrm{a}}}$ & 1.72 & 0.5 & - & - & $190 \pm 0.83$ & $0.35 \pm 0.01$ & $-30.42 \pm 2.9 \mid$ & & \\
\hline $\mathrm{F}^{\mathrm{a}}$ & 1.76 & 0.5 & - & - & $220 \pm 0.9 \mid$ & $0.42 \pm 0.04$ & $-32.01 \pm 3.21$ & & \\
\hline F3 & 1.75 & 0.5 & - & - & $167 \pm 0.25$ & $0.22 \pm 0.01$ & $-34.85 \pm 2.03$ & & \\
\hline F4 & 1.25 & 0.5 & - & - & $190 \pm 1.50$ & $0.26 \pm 0.01$ & $-32.45 \pm 2.94$ & & \\
\hline F5 & 2.5 & 0.5 & - & - & $155 \pm 0.93$ & $0.18 \pm 0.05$ & $-31.69 \pm 1.57$ & & \\
\hline F6 & 1.75 & 0.25 & - & - & $218 \pm 1.02$ & $0.35 \pm 0.01$ & $-31.44 \pm 2.03$ & & \\
\hline F7 & 1.75 & I & - & - & $150 \pm 1.22$ & $0.14 \pm 0.01$ & $-33.91 \pm 3.12$ & & \\
\hline F8 & 1.75 & 0.5 & 10 & 10 & $|68 \pm 0.5|$ & $0.22 \pm 0.01$ & $-39.34 \pm 1.15$ & $90 \% \pm 2.03 \%$ & $50 \% \pm 1.65 \%$ \\
\hline $\mathrm{Fg}$ & 1.75 & 0.5 & 10 & 10 & $176 \pm 0.16$ & $0.20 \pm 0.01$ & $+48.05 \pm 3.12$ & $98 \% \pm 2.01 \%$ & $80 \% \pm 1.01 \%$ \\
\hline $\mathrm{FIO}$ & 1.75 & 0.5 & 10 & 10 & $173 \pm 0.26$ & $0.19 \pm 0.01$ & $+58.03 \pm 1.12$ & $98 \% \pm 2.01 \%$ & $95 \% \pm 3.01 \%$ \\
\hline
\end{tabular}

Notes: All formulations were prepared using GMO $2.5 \%(\mathrm{w} / \mathrm{v})$ with respect to total dispersion volume. ${ }^{\mathrm{a} F I}$ and F2 contain propylene glycol and PEG 400 , respectively. ${ }^{\mathrm{b}} \mathrm{F} 9$ and $\mathrm{F} 10$ contain ion-paired PMX at molar ratio of PMX:CTAB ( $\mathrm{I}: 2$ and $\mathrm{I}: 4$, respectively).

Abbreviations: CTAB, cetyltrimethylammonium bromide; \%EE, \% entrapment efficiency; GMO, glyceryl monooleate; LCNPs, liquid crystalline nanoparticles; PDI, polydispersity index; PMX, pemetrexed; P407, poloxamer-407; RSV, resveratrol.

of the prepared formulations was investigated (F1-F3). The concentration of hydrotropes represented $70 \% \mathrm{w} / \mathrm{w}$ of the amount of GMO. ${ }^{47}$ As summarized in Table 1, ethanol (F3) possessed a great tendency in reducing the viscosity of GMO which in turn facilitates prompt diffusion of the hydrotropic mixture into aqueous phase. This was evidenced by significant $(P<0.05)$ decrease in both PS and PDI compared to (F1, propylene glycol; F2, PEG 400). Therefore, ethanol was selected as the suitable hydrotrope for further investigations.

\section{Effect of ethanol concentration}

The influence of other ethanol concentrations (F4, 50; F5, $100 \% \mathrm{w} / \mathrm{w}$ relative to total lipid) on PS and PDI was investigated. As summarized in Table 1, it was obvious that increasing solvent concentration was associated with significant reduction in PS and PDI $(P<0.05)$. Increasing ethanol concentration beyond $70 \% \mathrm{w} / \mathrm{w}$ revealed insignificant decrease $(P>0.05)$ in the PS. Therefore, ethanol at the concentration of $70 \% \mathrm{w} / \mathrm{w}$ was chosen as an optimum hydrotrope concentration which resulted in promising PS of $167 \mathrm{~nm}$ and PDI of 0.2 (F3).

\section{Effect of stabilizer concentration}

P407 is a triblock copolymer widely used in stabilizing LCNPs. This hydrophilic nonionic surfactant has a unique capability in adsorbing to the surface of LCNPs, thus inhibiting their aggregation. In addition to its great tendency in stabilizing internal structure of LCNPs through preventing transition to other mesophase structures, herein, the influence of three different concentrations of P407 (0.25\%, 0.5\%, and $1 \% \mathrm{w} / \mathrm{v}$ ) was screened (F3, F6-F7). As summarized in Table 1, increasing P407 concentration from $0.25 \%$ to $0.5 \% \mathrm{w} / \mathrm{v}$ relative to total dispersion volume was associated with obvious reduction in PS and PDI. Increasing P407 concentration beyond $0.5 \% \mathrm{~W} / \mathrm{v}$ resulted in insignificant reduction in PS (F3, $167 \mathrm{~nm}$; F7, $150 \mathrm{~nm}$ ). Therefore, 0.5\% w/v P407 concentration was selected as the optimum concentration for additional investigations. Our results were in good agreement with Freag et $\mathrm{al}^{47}$ who reported that $0.5 \% \mathrm{w} / \mathrm{v} \mathrm{P} 407$ concentration relative to total dispersion volume was quite sufficient to stabilize LCNPs and maintain their internal structure.

\section{Effect of percentage drug loading}

In an attempt to minimize the dose of cytotoxic PMX and reduce its associated adverse effects, PMX and RSV were successfully loaded within LCNPs at the weight ratio of 1:1. The characteristic absorption bands of PMX and RSV (Figure S2) on the validated HPLC analysis (Table S1) with good linearity ranging from 0.4 to $2 \mathrm{mg} \% \mathrm{PMX}$ and RSV concentration (Figure S1) proved that both PMX and RSV were successfully loaded into LCNPs and the drug carrier was effective for the co-delivery of the two drugs. It was obviously observed that CTAB modification significantly increased \%EE of PMX (Table 1). The average \%EE of PMX and RSV in the optimized ion-paired nanoformulation was $95 \%$ and $98 \%$, respectively. Physicochemical characterization results indicated that loading RSV and PMX did not affect the physicochemical properties of LCNPs; it was found that PMX-RSV-loaded LCNPs possess almost similar PS and PDI to blank LCNPs.

\section{Zeta potential}

All LCNPs that were formulated without CTAB (F1-F8) showed a negatively charged zeta potential around $-34 \mathrm{mV}$ although GMO is a neutral lipid, which could be explained by adsorption of free hydroxyl groups on the surface of 
LCNPs in addition to free oleic acid found in commercially available GMO. ${ }^{48}$ A significant alteration in zeta potential upon the addition of CTAB was detected. As summarized in Table 1, ion-paired PMX-RSV-LCNPs (F9-F10) displayed a positively charged zeta potential which is sufficient for the colloidal stability and preventing aggregation.

\section{Phase behavior}

Polarized light microscope is commonly utilized to study the liquid crystalline phases depending on their textures. The hexagonal phases exhibit a fan-like texture, whereas the cubic phases show a dark diffraction pattern when viewed under a polarizing light microscope. Herein, both blank and PMX-RSV-loaded LCNPs demonstrated a dark diffraction patterns when examined under polarizing microscope reflecting the isotropic nature of the formulations. ${ }^{41}$

\section{Transmission electron microscopy}

The surface morphology of optimized ion-paired PMX-RSVLCNPs was visualized via TEM. As depicted in Figure 1A,
LCNPs displayed spherical particles with an average PS of $180 \mathrm{~nm}$ which was in agreement with the measurements obtained by DLS.

\section{In vitro release study}

In vitro release behavior of each free RSV, free PMX, PMX-RSV-LCNPs (F8), and ion-paired PMX-RSV-LCNPs (F9-F10) was examined in commonly utilized release media PBS ( $\mathrm{pH}=7.4)$. As shown in Figure 1B, both free RSV and PMX exhibited burst release profile, a relatively rapid and entire release was observed after 2 and 4 hours for PMX and RSV, respectively. However, after incorporation of drugs into LCNPs, a sustained release behavior of both drugs was observed. The release behavior of RSV was characterized by a biphasic release pattern where a relatively initial burst release within first 3-4 hours was observed followed by sustained release up to 24 hours. The initial burst release could be explained via relatively large surface area-to-volume ratio provided by LCNPs, while the continuous sustained release of drugs from PMX-RSV-LCNPs could be referred to the

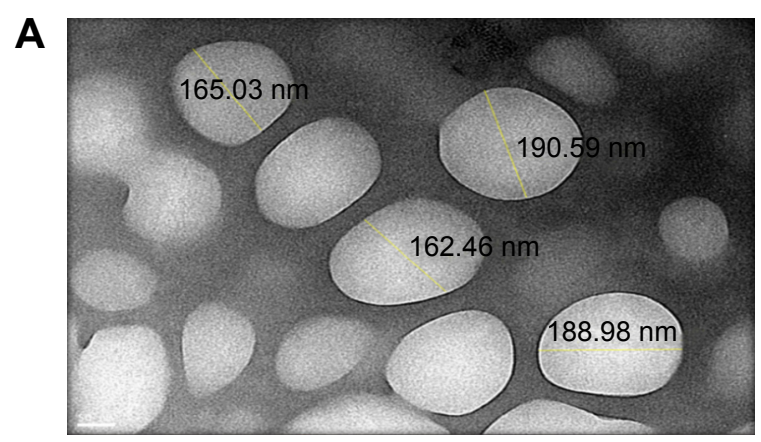

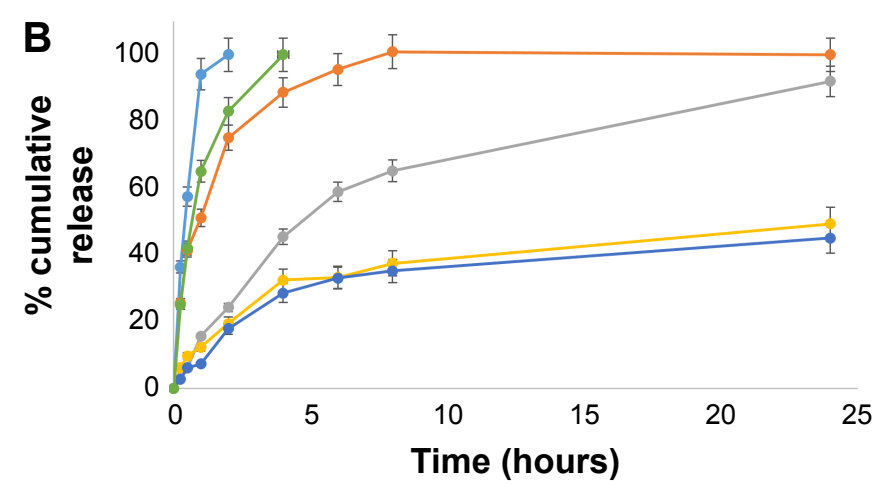

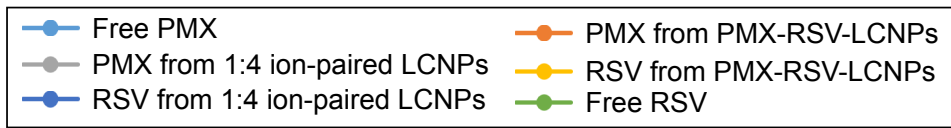

C 100

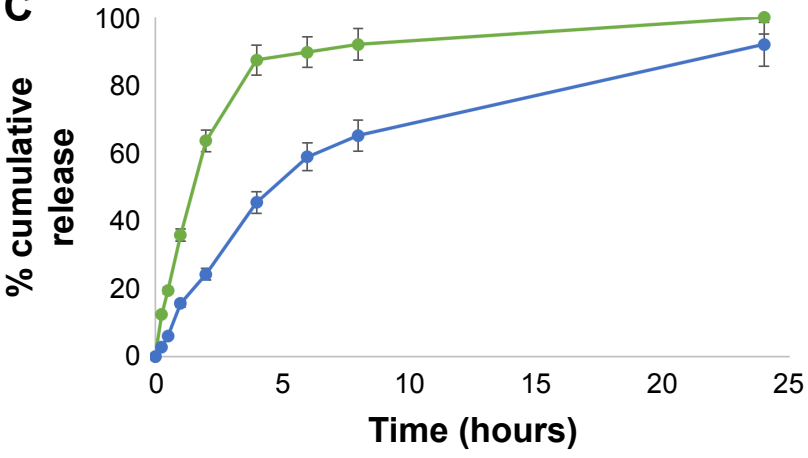

Figure I Physicochemical characterization of optimized LCNPs.

Notes: (A) Transmission electron micrograph displaying morphology of ion-paired PMX-RSV-LCNPs (FI0). (B) In vitro release of PMX and RSV from PMX-RSV-LCNPs (F8) and ion-paired PMX-RSV-LCNPs (FI0) in comparison to free PMX and RSV solution in PBS (pH 7.4) at 100 rpm and $37^{\circ} \mathrm{C}$ using the dialysis bag diffusion method. (C) The influence of different CTAB:PMX molar ratios on the release of PMX from ion-paired LCNPs in PBS (pH 7.4) at $100 \mathrm{rpm}$ and $37^{\circ} \mathrm{C}$ using the dialysis bag diffusion method. Abbreviations: CTAB, cetyltrimethylammonium bromide; LCNPs, liquid crystalline nanoparticles; PMX, pemetrexed; RSV, resveratrol. 


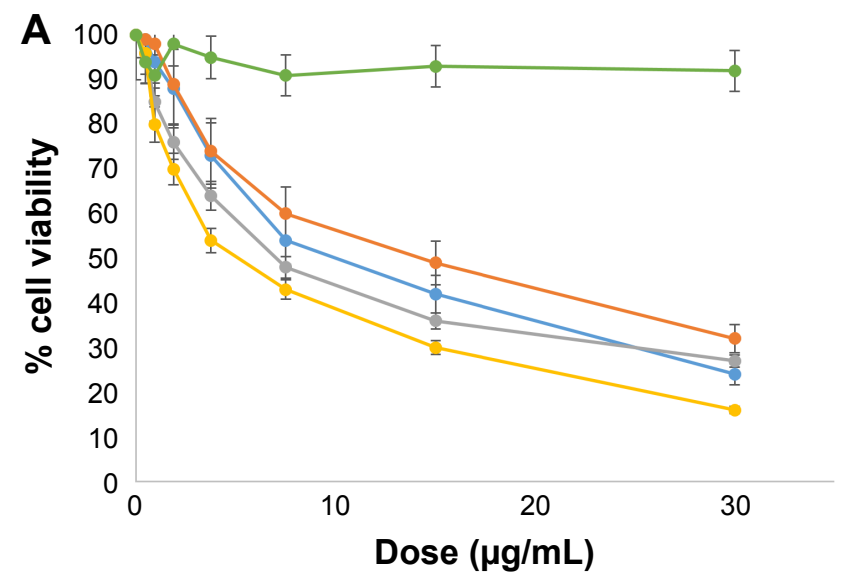

\begin{tabular}{ll|}
$-\rightarrow$ Free RSV & - Free PMX \\
- Free PMX/RSV & - PMX-RSV-LCNPs \\
- Blank LCNPs & \\
\hline
\end{tabular}

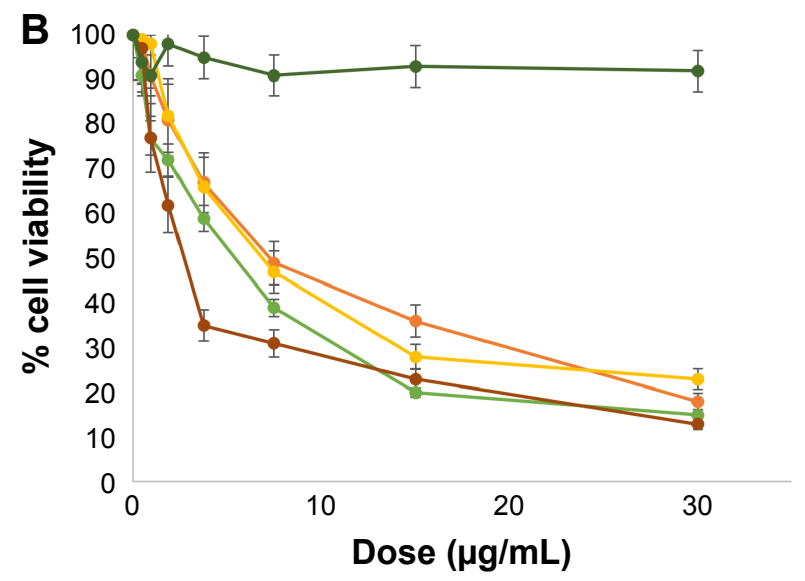

$\begin{array}{ll}\longrightarrow \text { Free RSV } & - \text { Free PMX } \\ \multimap \text { Free PMX/RSV } & - \text { PMX-RSV-LCNPs } \\ \rightarrow \text { Blank LCNPs } & \end{array}$

Figure 2 In vitro cytotoxicity of ion-paired PMX-RSV-LCNPs (FI0) compared to blank LCNPs, free PMX, free RSV, and free PMX/RSV cosolvent on A549 NSCLC cell line at the concentration of $0.468-30 \mu \mathrm{g} / \mathrm{mL}$ expressed as \% cell viability after (A) 24 hours and (B) 48 hours.

Abbreviations: LCNPs, liquid crystalline nanoparticles; NSCLC, non-small-cell lung cancer; PMX, pemetrexed; RSV, resveratrol.

diffusion of both drugs from the lipid core of bicontinuous bilayer of the liquid crystalline structure. ${ }^{49}$ To check the effect the HIP process on the release behavior of PMX (Figure 1C), two formulations of modified PMX-RSV-LCNPs with two different concentrations of CTAB (F9-F10) were compared to unmodified PMX-RSV-LCNPs (F8). Compared to unmodified LCNPs, CTAB-modified LCNPs possess an additional controlled release of PMX and upon increasing the concentration of CTAB (F10), more sustained release behavior from regular lipid channels was observed. This may be attributed to the extra hydrophobic nature imparted to LCNPs by CTAB. Obviously, the proposed ion-paired PMX-RSV-LCNPs provided an acceptable sustained release pattern of PMX up to 24 hours. This sustained drug release behavior is an essential prerequisite to guarantee constant and controlled release of anticancer drugs within tumor cells which in turn reduce their systemic cytotoxicity.

\section{In vitro cytotoxicity studies}

The cytotoxic influence of free RSV, free PMX, free RSV/ PMX mixture, blank LCNPs, and ion-paired PMX-RSVLCNPs (F10) on A549 lung cancer cell line was determined using MTT assay (Figure 2A and B). Better therapeutic outcomes for resistant tumor cells were mainly achieved via combinational therapy based on two or more anticancer agents. Coadministering of herbal drugs with potent anticancer drugs hold great promise, particularly, PMX and RSV have been reported for their in vitro cytotoxic activity on lung cancer cells. ${ }^{27}$ Furthermore, the combined doses of PMX and RSV loaded into PMX-RSV-LCNPs were precisely determined by their dose dependency and relative amount of PMX vs RSV to A549 cells, to maximize the synergistic antitumor efficacy. Blank LCNPs demonstrated good cytocompatibility with insignificant cytotoxicity on A549 cancer cells after 24 hours (Figure 2A) and 48 hours (Figure 2B; \% cell viability $>85 \%$ ). RSV and PMX were evaluated at concentration ranged from 0.468 to $30 \mu \mathrm{g} / \mathrm{mL}$. Free PMX and RSV exhibited a dosedependent cytotoxic efficacy on the lung cancer cells. To check the synergistic activity between both drugs, the combined $\mathrm{IC}_{50}$ was calculated and is summarized in Table 2. As summarized in the table, free RSV/PMX mixture exhibited a lower $\mathrm{IC}_{50}$ value $(4.5 \mu \mathrm{g} / \mathrm{mL})$ compared to each free PMX and free RSV ( $\mathrm{IC}_{50}: 7.9$ and $8.4 \mu \mathrm{g} / \mathrm{mL}$, respectively). It was clearly observed that PMX-RSV-LCNPs had a significant superior cytotoxic activity on lung cancer cells compared to free RSV/PMX mixture. This superiority in the cytotoxicity of LCNP formulations could be attributed to the enhanced internalization of LCNPs facilitated by both bioadhesive property of GMO and energy-independent (cholesterol-dependent)

Table $2 \mathrm{IC}_{50}$ of free drugs and optimized LCNPs after 24 and 48 hours using Compusyn ${ }^{\circledR}$ software

\begin{tabular}{l|l|l|l|l}
\hline $\begin{array}{l}\text { IC }_{50} \\
(\mu \mathrm{g} / \mathrm{mL})\end{array}$ & $\begin{array}{l}\text { Free } \\
\text { PMX }\end{array}$ & $\begin{array}{l}\text { Free } \\
\text { RSV }\end{array}$ & $\begin{array}{l}\text { Free } \\
\text { RSV/PMX }\end{array}$ & $\begin{array}{l}\text { Ion-paired } \\
\text { PMX-RSV-LCNPs }\end{array}$ \\
\hline After 24 hours & 10.10 & 12.66 & 7.96 & 5.50 \\
\hline After 48 hours & 7.94 & 8.43 & 4.51 & 4.0628 \\
\hline
\end{tabular}

Abbreviations: LCNPs, liquid crystalline nanoparticles; PMX, pemetrexed; RSV, resveratrol. 
uptake mechanism associated with LCNPs resulting into higher intracellular concentration of both RSV and PMX. ${ }^{50}$ It is worth to mention that the cytotoxicity of PMX-RSV-LCNPs was more evident following 48 -hour incubation than 24 hours which could be ascribed to the sustained release behavior of RSV and PMX imparted by LCNPs.

\section{Cellular uptake of optimized LCNPs}

The qualitative cellular uptake study of the proposed LCNPs was carried out on A549 cell line using confocal microscopy. Coumarin-6 was used as a model hydrophobic fluorescent dye which could be easily entrapped within lipid bilayer of LCNPs. To assess the entrapment and retention of dye in LCNPs, the coumarin-6-loaded LCNPs were placed in centrifugal ultrafilter (Vivaspin ${ }^{\circledR}$ 6, molecular weight cutoff [MWCO]100 kDa; Vivaproducts, Inc, Littleton, MA). The ultrafilters were then centrifuged at $4,000 \mathrm{rpm}$ for 30 minutes. The fraction of dye released (unentrapped) during 24 hours with all formulations was within $1 \%-2 \%$, suggesting a good entrapment. Untreated A549 cells were used to exclude cellular autofluorescence. A549 cells were incubated with free coumarin-6 and coumarin-6-loaded LCNPs for 4 (Figure 3A) and 24 hours (Figure 3B). As shown in the figure, LCNPs demonstrated superior cellular uptake compared to free dye. The increased intracellular concentration after 24 hours confirmed the time-dependent cellular uptake of LCNPs. To guarantee that ion-paired LCNPs were taken up and internalized into A547 and not only adsorbed onto the

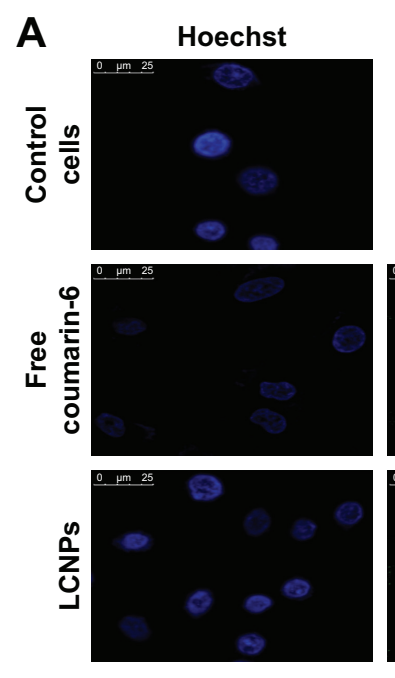

Flouresence

Merge

DIC
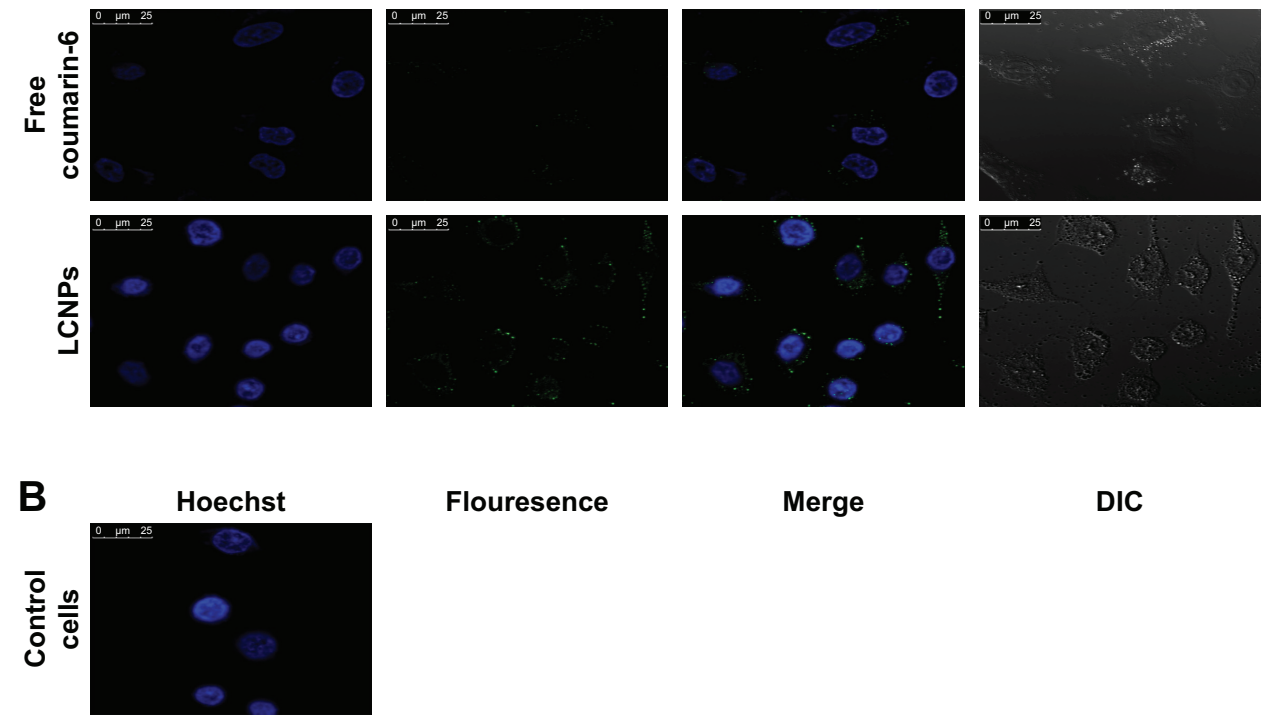

Flouresence

Merge

DIC
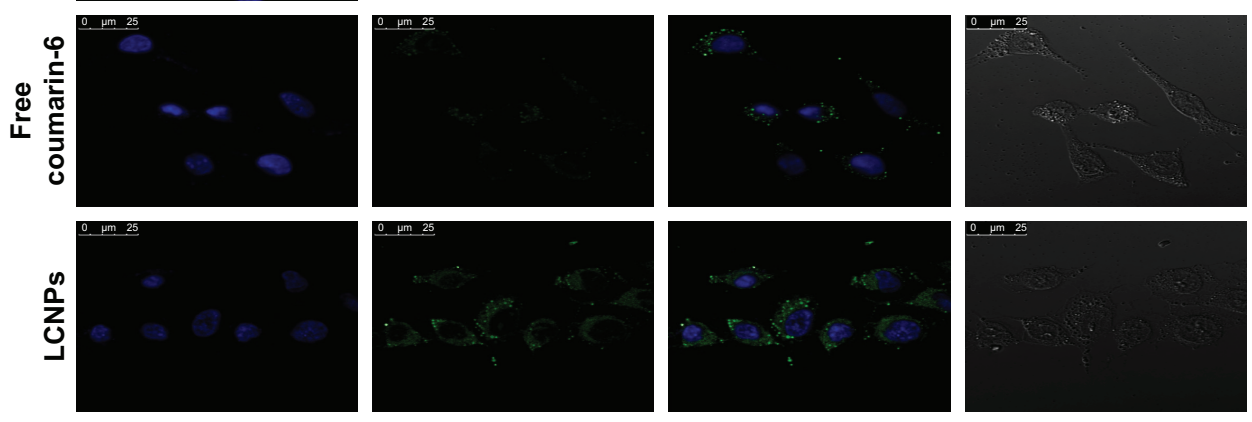

Figure 3 Confocal microscopy images demonstrating cellular uptake of coumarin-6-loaded LCNPs relative to free coumarin-6 and control cells after incubation with A549 lung cancer cell line for (A) 4 hours and (B) 24 hours in which the first channel of nuclei is represented by blue stain of Hoechst, the second channel of free coumarin-6 or coumarin-6-loaded LCNPs is represented by green fluorescence, third channel of merge is represented by overlay between two channels, and finally the fourth channel of DIC is represented by the light microscopic image of A549 cells.

Abbreviations: LCNPs, liquid crystalline nanoparticles; DIC, differential interference contrast. 
surface of cellular membrane, their uptake was evaluated using z-stack images. The z-direction image (Figure S3) displayed the cross-sectional uptake of coumarin-6-loaded LCNPs in which they were traveled from the apical surface of cellular membrane toward the basolateral membrane represented from z-0 to z-29. Image of z-29 represents maximum intensity projections of whole A549 cells. The fluorescence images manifested that LCNPs with a size of $173 \mathrm{~nm}$ move rapidly across the cellular membrane, distribute within the cytosol, successively move across different cellular planes, and localize in the perinuclear region. The video displaying z-stacks confirmed particle localization within the cells mainly within the cytoplasm and seldom dispersed in the nuclei was included as provided in Supplementary materials. The enhanced cellular uptake of coumarin-6-loaded LCNPs could be attributed to GMO bioadhesive and membranefusing features in addition to the unique structure of LCNPs in which water channels surrounded by bicontinuous lipid bilayer offer hydrophilic-hydrophobic pattern which facilitates interaction with hydrophobic cholesterol on cell surface. ${ }^{50}$ In addition, it is worth mentioning that nanoparticle size, shape, and surface charge could possibly affect their interaction with the cellular membrane. It was previously reported that NPs with an average size between 200 and $350 \mathrm{~nm}$ could successfully distribute to different tissues, whereas NPs $<70 \mathrm{~nm}$ were instantly excreted by the kidneys. Moreover, the positively charged surface imparted by CTAB could further facilitate cellular uptake by binding to the anionic surface proteoglycans via adsorptive-mediated endocytosis. ${ }^{51}$ On the other hand, free coumarin-6 entered the cell via simple diffusion resulted in rapid saturation of intracellular region prohibiting further entry of free dye by time unlike LCNPs which entered via endocytosis resulted in gradual release of free dye thus evading intracellular saturation. These findings were in accordance with MTT assay in which optimized PMX/RSV-loaded LCNPs displayed higher cytotoxic effect than free PMX/RSV mixture.

\section{In vivo antitumor activity}

The purpose of this study was to investigate the antitumor efficacy and systemic toxicity of the synergistic combination (PMX and RSV) administered intravenously either in free form or loaded within the optimized LCNPs. This study was carried out over 25 days on Balb/c male urethane-induced lung cancer-bearing mice. The antitumor efficacy of PMXRSV-LCNPs (F10) was evaluated via both macroscopic and microscopic examination after lung cancer induction and/or PMX-RSV-LCNP treatment. The average lung weight of tumor-bearing mice treated with free PMX/RSV mixture
(380 mg \pm 10$)$ was significantly lower than the positive control group (605 \pm 25$)(P<0.05)$. However, the mice treated with PMX-RSV-LCNPs showed the best reduction in average lung weight ( $260 \pm 15 \mathrm{mg})$ with insignificant difference to that of the negative control group (195 $\pm 10 \mathrm{mg})$, confirming the great potentiality of optimized ion-paired PMX-RSV-LCNPs (F10) in improving the therapeutic outcomes (Figure 4A). Figure 4B shows the representative images of excised lungs from different mice groups where excised lungs of positive control group showed malignant surface lesions characterized by a massive number of lung adenomatous foci, while lungs obtained from PMX-RSV-LCNP (F10)-treated group significantly possess normal physiological features with significantly reduced malignant surface lesions.

\section{Tumor biomarkers}

To determine the therapeutic efficacy of intravenously administered free PMX/RSV mixture and optimized ion-paired PMX-RSV-LCNPs (F10), tumor biomarkers were assessed. As depicted in Figure 4C, the level of VEGF in lungs treated with ion-paired PMX-RSV-LCNPs (F10; 3.5 pg/g) was significantly $(P<0.05)$ lower than those treated with free PMX/ RSV mixture $(5.21 \mathrm{pg} / \mathrm{g})$. The FDA-approved multi-targeted antifolate PMX plays a major role in decreasing growth rate of tumor cells which subsequently minimizes the need for vascularization and angiogenesis and decreases the tumor burden. ${ }^{52}$ Moreover, the chemopreventive polyphenolic RSV was reported as antiangiogenic factor which reduces VEGF secretion from cocultured A549. ${ }^{53}$ Thanks to the synergistic effect of our chemo-herbal combination (PMX-RSV), the developed nanocarriers succeeded to develop additional control for tumor growth. ${ }^{27}$

These results were in agreement with CASP-3 level (Figure 4D) that is activated in the apoptotic cells in which lungs treated with ion-paired PMX-RSV-LCNPs (F10) showed significantly higher active CASP-3 $(2.8 \mathrm{ng} / \mathrm{g}$ tissue) than those treated with free PMX/RSV (1.9 ng/g tissue) $(P<0.05)$.

\section{Histopathological and immunohistochemical analysis}

As depicted in Figure 5A, the H\&E-stained lung sections revealed normal physiological features of negative control mice in which normal alveoli were lined with single epithelial tissues, whereas the positive urethane-treated control group exhibited various preneoplastic to neoplastic lesions extending from epithelial hyperplasia to adenoma (blue arrow). In addition to hemorrhage and inflammatory cells, infiltration 
A

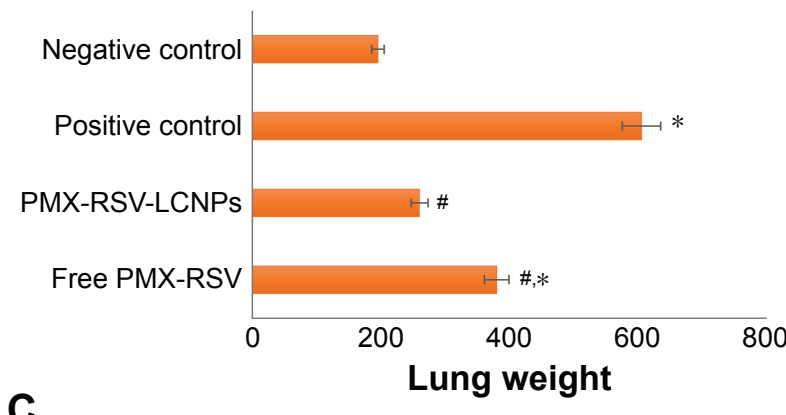

C

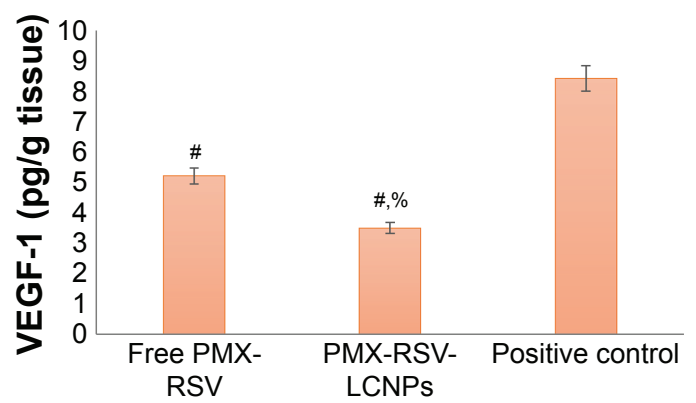

B

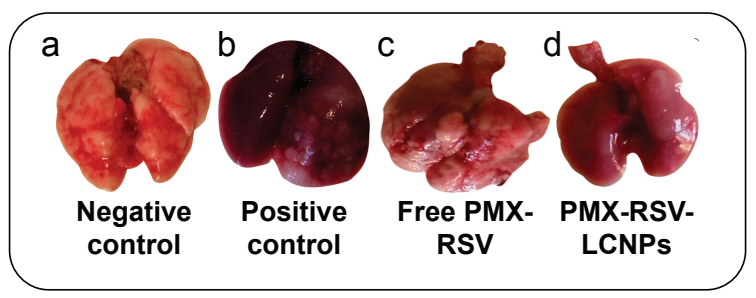

D

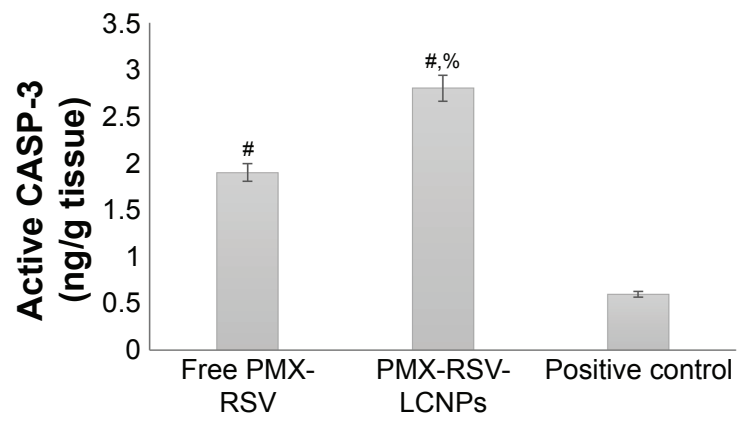

Figure 4 In vivo antitumor activity.

Notes: (A) Average weight of excised lungs of mice measured at the end of treatment course. (B) Representative images of excised lung tumors displaying differences among mice groups after 3 weeks of exposing to different treatments; (a) negative control, (b) positive control, (c) free PMX-RSV mixture, and (d) ion-paired PMX-RSVLCNPs (FIO). The level of the tumor biomarkers measured using ELISA in tumor homogenate including (C) VEGF and (D) CASP-3 in urethane-induced lung cancer-bearing mice treated with free PMX-RSV and ion-paired PMX-RSV-LCNPs (FI0) compared to untreated positive control. *P<0.05 vs negative control, $\# P<0.05$ vs positive control, $\% P<0.05$ vs free $P M X / R S V$ mixture.

Abbreviations: CASP-3, caspase 3; LCNPs, liquid crystalline nanoparticles; PMX, pemetrexed; RSV, resveratrol; VEGF, vascular endothelial growth factor.

represented by white and black, respectively. Free PMX/RSV mixture-treated mice were associated with reduction in histopathological profile of neoplastic transformation, while the ion-paired PMX-RSV-LCNP (F10)-treated group displayed an outstanding amelioration in the carcinogenic histopathological profile. It was observed that, compared to the positive control groups, lungs harvested from lung cancer-bearing mice treated with IV injection of free PMX/RSV mixture

A
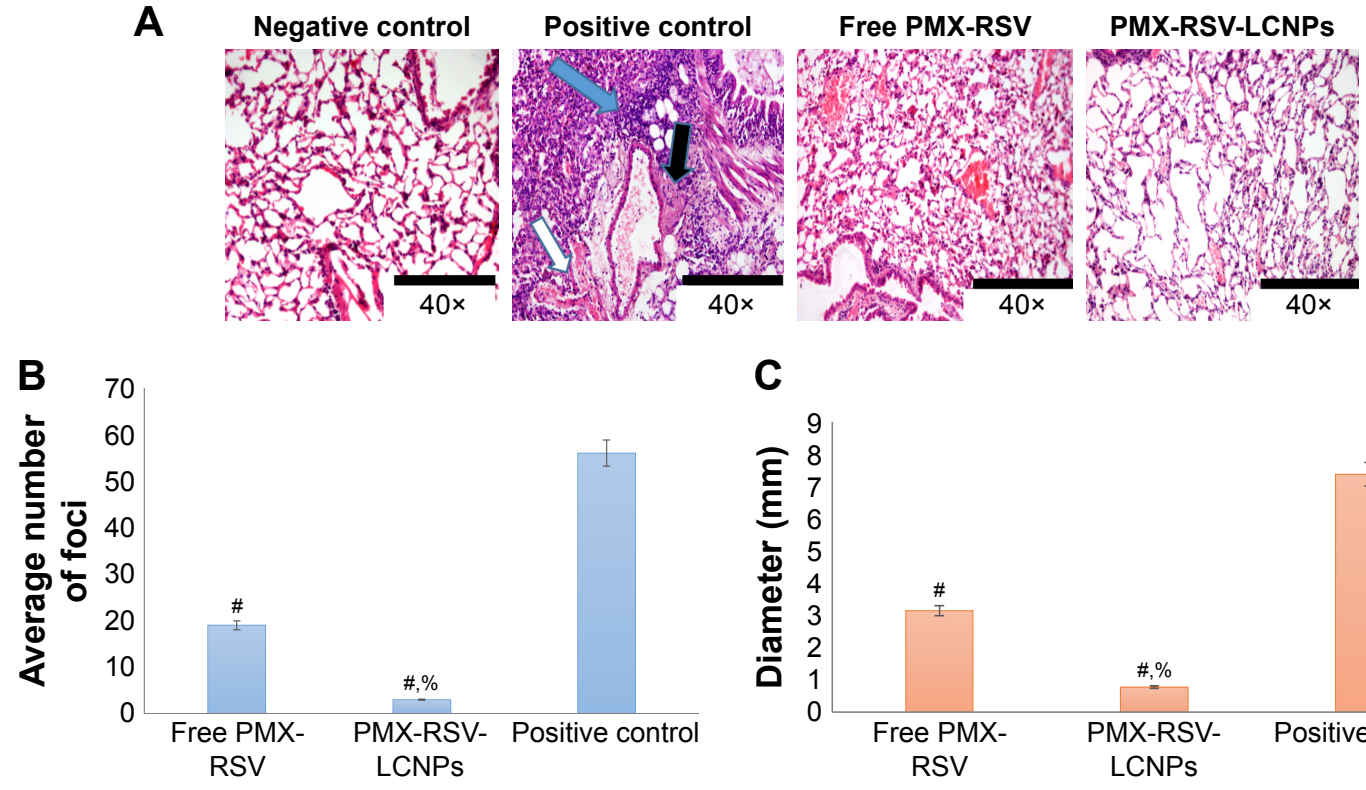

C

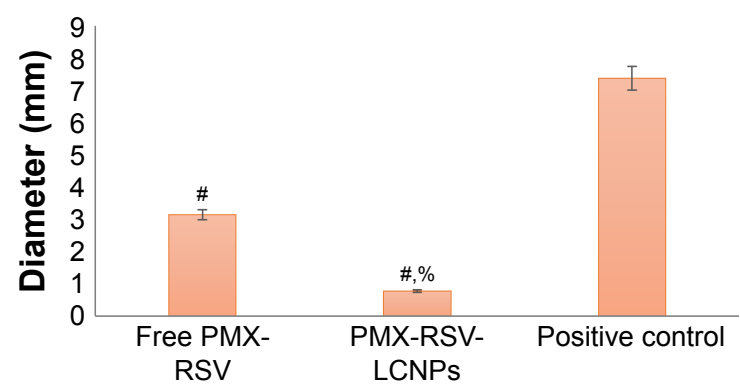

Figure 5 (A) Histopathological analysis of H\&E-stained excised lung sections of mice treated with free PMX-RSV and ion-paired PMX-RSV-LCNPs (FI0) in comparison to positive control and negative control groups. (B) Average number of foci in the excised lungs, (C) The diameter of lung foci ( $\mathrm{mm}$ ).

Notes: ${ }^{\# P}<0.05$ vs positive control, $\%$ P $<0.05$ vs Free PMX/RSV mixture.

Abbreviations: LCNPs, liquid crystalline nanoparticles; PMX, pemetrexed; RSV, resveratrol. 
resulted in the reduced number of adenomatous foci while those harvested from mice treated systemically with optimized ion-paired PMX-RSV-LCNPs (F10) displayed the best reduction in both number and diameter of lung adenomatous foci (Figure 5B and C). Furthermore, the immunohistochemical analysis revealed the reduced expression of the proliferation marker Ki-67 protein indicating the superiority of ion-paired PMX-RSV-LCNPs (F10) in attenuating proliferation of urethane-induced adenomas (Figure 6A and B).

\section{Safety index}

It was observed that positive control mice suffered from gradual loss in body weight of along the whole experimental duration which could be attributed to lung tumor invasion and malfunction. Compared to the free combined PMX/ RSV group, the PMX-RSV-LCNP (F10) group showed less changes in total body weight which suggested lower adverse effects of PMX-RSV-LCNPs (Figure 7A). Strikingly, PMX-RSV-LCNP (F10)-treated mice maintained the well-organized alveolar structure of lung tissues which significantly improved their survival rates up to $60 \%$ during the experimental period compared to only $30 \%$ survival rate of mice treated with free PMX/RSV mixture (Figure 7B).
The death of mice was based on remarkable disruption of alveolar structure resulted from lung damage of urethanetreated mice. ${ }^{54,55}$

PMX is well known for its hepato- and nephrotoxicity. However, RSV plays an important role in attenuating toxicity associated with PMX as it exerts hepato- and nephroprotective effect. ${ }^{56-59}$ Our proposed LCNPs enabled the delivery of the synergistic combination of RSV and PMX which played an important role in improving treatment outcomes based on their capability in killing cancerous cells in addition to remarkable reduction in the dosages of PMX which alleviates the toxicity concerns of the proposed PMX-RSV-LCNP formulation at the systemic level. Compared to untreated positive control or mice treated with free PMX/RSV mixture, optimized nanoformulation induced the least damage to the kidney and liver as demonstrated in Figure 7C and D, respectively. Conclusively, the improved therapeutic outcomes of urethane-induced lung cancer-bearing mice receiving PMXRSV-LCNPs could be attributed to the great tendency of LCNPs in protecting these payloads from premature release in blood stream and reaching other organs which in turn maximize accumulation of synergistic combination (PMX/RSV) in cancer tissue. In addition, the leaky vasculature within
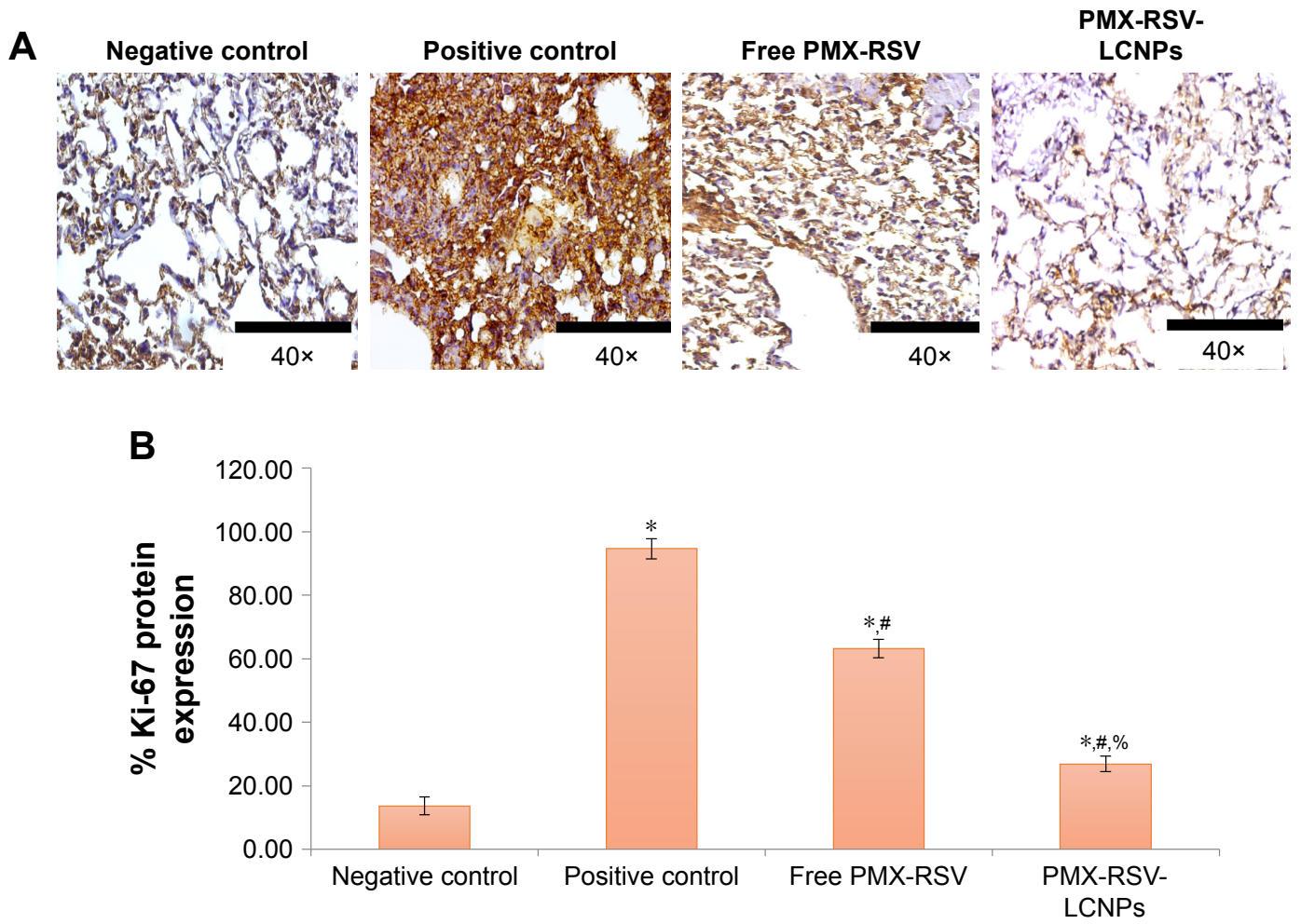

Figure 6 Immunohistochemical analysis: (A) Ki-67 staining. (B) \% expression of Ki-67 of excised lung sections of urethane-induced lung cancer-bearing mice received free PMX-RSV and ion-paired PMX-RSV-LCNPs (FI0) in comparison to untreated positive control.

Note: $* P<0.05$ vs negative control, ${ }^{*} P<0.05$ vs positive control, $\% P<0.05$ vs free $P M X-R S V$ mixture.

Abbreviations: LCNPs, liquid crystalline nanoparticles; PMX, pemetrexed; RSV, resveratrol. 
A
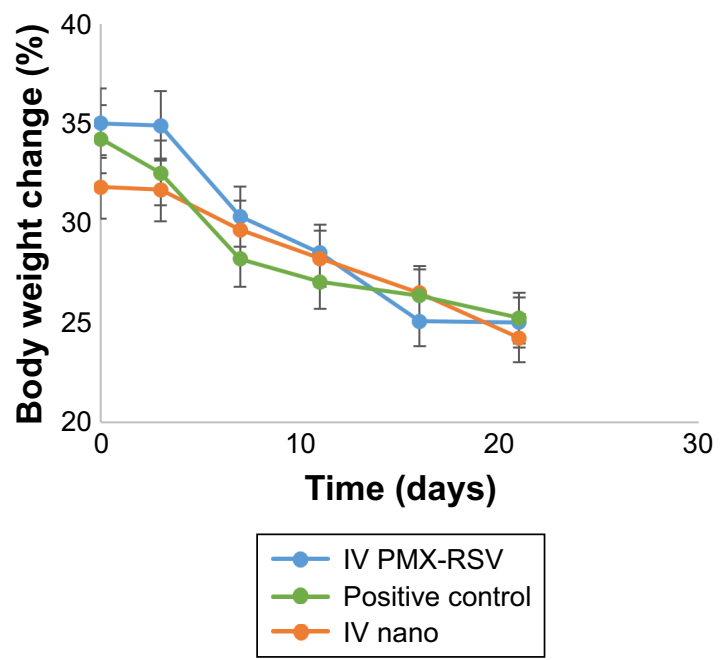

C

D
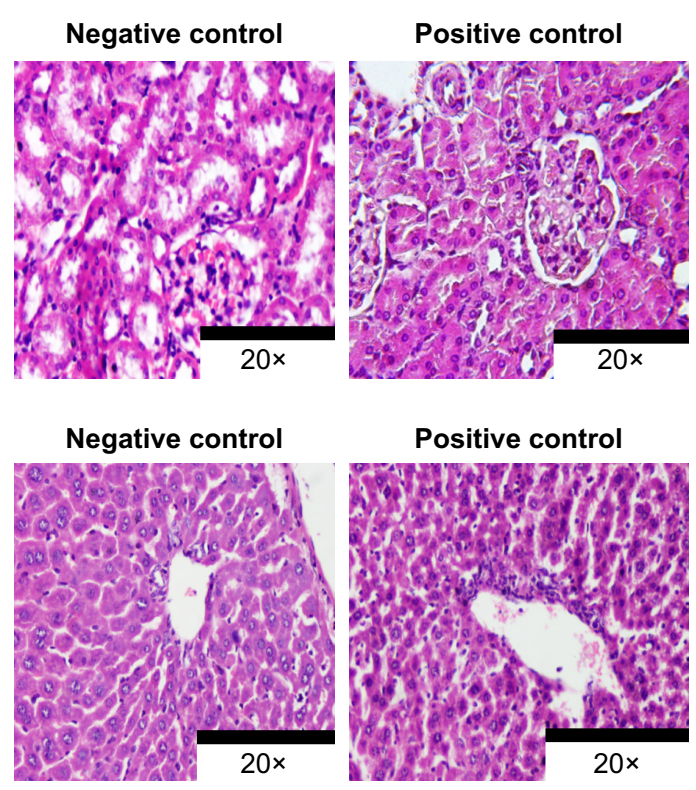

B

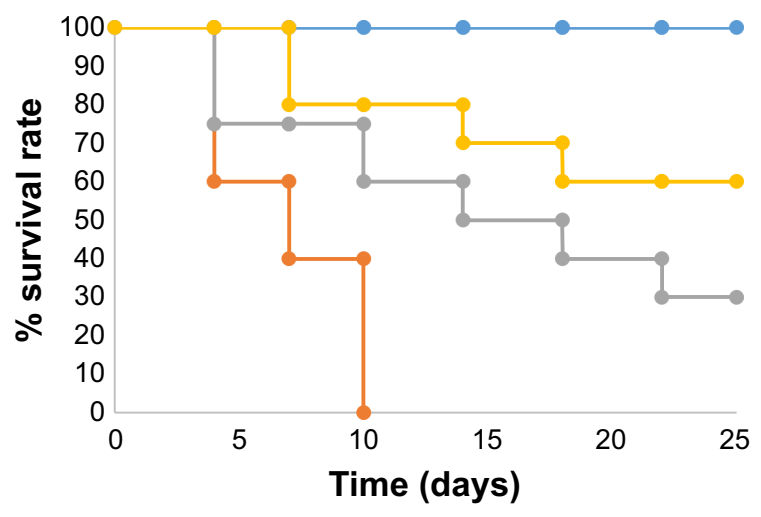

Positive control

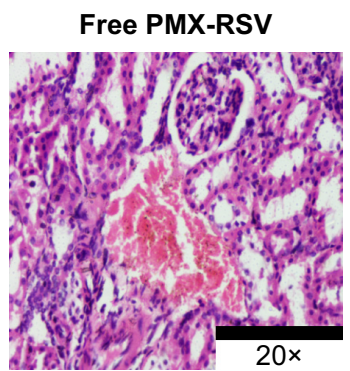

Free PMX-RSV

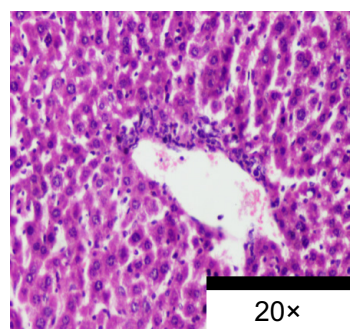

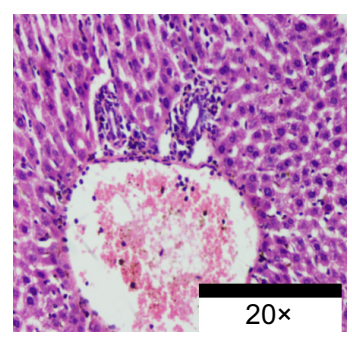

PMX-RSV-LCNPs

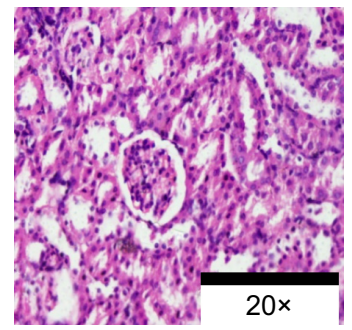

PMX-RSV-LCNPS

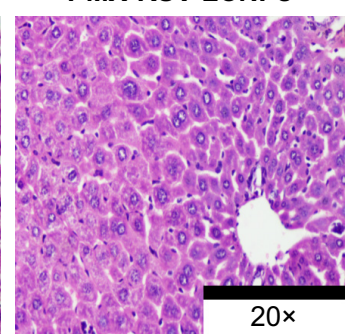

Figure 7 Assessment of safety index of optimized LCNPs.

Notes: (A) Average body weights of urethane-induced lung cancer-bearing mice treated with free PMX-RSV and ion-paired PMX-RSV-LCNPs (FI0) for 3 weeks compared to untreated positive control. (B) Survival rate curve among treated groups in comparison to positive and negative control groups. H\&E-stained kidney (C) and liver (D) of different treatment groups.

Abbreviations: IV, intravenous; LCNPs, liquid crystalline nanoparticles; PMX, pemetrexed; RSV, resveratrol.

tumor site and impaired lymphatic drainage facilitate penetration and retention of the proposed LCNPs with nanometric PS of $173 \mathrm{~nm}$. Obviously, PMX-RSV-LCNPs could efficiently target and suppress lung cancers which in turn enhanced survival rates and remarkably minimized systemic side effects as compared to free PMX/RSV.

\section{Conclusion}

In this study, we shed light on the potential of LCNPs for the co-delivery of promising synergistic combination of
FDA-approved PMX and herbal RSV for lung cancer treatment. PMX-RSV-LCNPs were prepared via simple hydrotrope method. In situ HIP was carried out using CTAB to improve encapsulation efficiency and to guarantee extended release behavior of hydrophilic PMX up to 24 hours. This can interestingly minimize the dose of chemotherapeutic PMX and overcome MDR. The proposed dual drug-loaded LCNPs manifested promising cytotoxic effects as well as enhanced cellular uptake against A549 lung cancer cells. The superior in vivo antitumor efficacy of ion-paired PMX-RSV-LCNPs 
was demonstrated in terms of reduced lung weights, reduced number of lung adenomatous foci and their corresponding diameter, lower angiogenic biomarker (VEGF), and higher apoptotic biomarker (CASP-3). Furthermore, they are associated with an improved histopathological and immunohistochemical profile. Strikingly, the proposed system reduced hepato- and nephrotoxicity commonly associated with PMX. Collectively, the ion-paired PMX-RSV-LCNPs offer a promising avenue for lung cancer treatment via combining the merits of chemotherapeutic and herbal drugs.

\section{Summary points}

- This work has been directed to the preparation of LCNPs for the co-delivery of chemo-herbal drug combination of RSV and PMX for effective lung cancer treatment.

- In situ ion-pairing approach was utilized to alter the solubility characteristics of PMX and enhance its EE into the lipidic matrix. LCNPs co-loaded with RSV and ion-paired PMX were successfully tailored with PS around $173 \mathrm{~nm}$ and high $\%$ EE for both drugs.

- Sustained and continuous drug release behavior up to 24 hours was successfully achieved. Such sustained release pattern is preferred to decrease the systemic cytotoxicity associated with PMX.

- PMX-RSV-LCNPs demonstrated enhanced cytotoxicity on A549 lung cancer cell line as determined by MTT assay as well as superior cellular uptake attributed to the bioadhesive and membrane-fusing features of GMO.

- The in vivo antitumor efficacy of PMX-RSV-LCNPs was evaluated on lung cancer-bearing mice. PMX-RSVLCNPs showed a superior antitumor efficacy evidenced by reduced lung weights, diminished number of lung adenomatous foci as well as their diameter, lower level of the angiogenic biomarker (VEGF), and higher apoptotic biomarker (CASP-3) level.

- PMX-RSV-LCNPs demonstrated an improved histopathological and immunohistochemical profile compared to free drugs.

- In vivo toxicity study revealed that the proposed PMXRSV-LCNP system reduced hepato- and nephrotoxicity associated with free PMX.

- In summary, LCNPs gathered the privileges of both chemotherapeutic and herbal drugs which could offer a new potential avenue for lung cancer treatment.

\section{Acknowledgment}

This work was supported by the research grant (No 5731) from Science and Technology Development Fund (STDF), Ministry of Scientific Research, Egypt.

\section{Disclosure}

The authors report no conflicts of interest in this work.

\section{References}

1. Abdelaziz HM, Gaber M, Abd-Elwakil MM, et al. Inhalable particulate drug delivery systems for lung cancer therapy: nanoparticles, microparticles, nanocomposites and nanoaggregates. J Control Release. 2018; 269:374-392.

2. Smith RA, Andrews KS, Brooks D, et al. Cancer screening in the United States, 2017: a review of current American Cancer Society guidelines and current issues in cancer screening. CA Cancer J Clin. 2017;67(2): $100-121$.

3. American Cancer Society. What Is Non-Small Cell Lung Cancer? Atlanta, GA: American Cancer Society; 2016.

4. Zappa C, Mousa SA. Non-small cell lung cancer: current treatment and future advances. Transl Lung Cancer Res. 2016;5(3):288-300.

5. Midthun DE. Early detection of lung cancer. F1000Res. 2016;5:739.

6. American Cancer Society. Treatment Choices for Non-Small Cell Lung Cancer, by Stage. Atlanta, GA: American Cancer Society; 2017.

7. Chidambaram M, Manavalan R, Kathiresan K. Nanotherapeutics to overcome conventional cancer chemotherapy limitations. J Pharm Pharm Sci. 2011;14(1):67-77.

8. Chemotherapy And Selective Toxicity [webpage on the Internet]. All Answers Ltd.; 2013. Available from: https://www.ukessays.com/ essays/biology/chemotherapy-and-selective-toxicity-biology-essay. php? vref=1. Accessed December 28, 2018.

9. Elzoghby AO, Elgohary MM, Kamel NM. Implications of protein- and Peptide-based nanoparticles as potential vehicles for anticancer drugs. Adv Protein Chem Struct Biol. 2015;98:169-221.

10. Cohen MH, Johnson JR, Wang YC, Sridhara R, Pazdur R. FDA drug approval summary: pemetrexed for injection (Alimta) for the treatment of non-small cell lung cancer. Oncologist. 2005;10(6):363-368.

11. PubChem [homepage on the Internet]. Compound Database; $\mathrm{CID}=446556$. Available from: https://pubchem.ncbi.nlm.nih.gov/ compound/446556. Accessed December 3, 2017.

12. Adjei AA. Pharmacology and mechanism of action of pemetrexed. Clin Lung Cancer. 2004;5(Suppl 2):S51-S55.

13. Chattopadhyay S, Moran RG, Goldman ID. Pemetrexed: biochemical and cellular pharmacology, mechanisms, and clinical applications. Mol Cancer Ther. 2007;6(2):404-417.

14. Fuld AD, Dragnev KH, Rigas JR. Pemetrexed in advanced non-smallcell lung cancer. Expert Opin Pharmacother. 2010;11(8):1387-1402.

15. Ho C, Davies AM, Sangha RS, et al. Phase I/II trial of pemetrexed plus nab-paclitaxel in advanced solid tumor patients with emphasis on non-small cell lung cancer. Invest New Drugs. 2013;31(6):1587-1591.

16. Mahmud F, Jeon OC, Alam F, et al. Oral pemetrexed facilitates low-dose metronomic therapy and enhances antitumor efficacy in lung cancer. J Control Release. 2018;284:160-170.

17. El-Far SW, Helmy MW, Khattab SN, Bekhit AA, Hussein AA, Elzoghby AO. Phytosomal bilayer-enveloped casein micelles for codelivery of monascus yellow pigments and resveratrol to breast cancer. Nanomedicine. 2018;13(5):481-499.

18. Kabary DM, Helmy MW, Elkhodairy KA, Fang JY, Elzoghby AO. Hyaluronate/lactoferrin layer-by-layer-coated lipid nanocarriers for targeted co-delivery of rapamycin and berberine to lung carcinoma. Colloids Surf B Biointerfaces. 2018;169:183-194.

19. Sabra SA, Elzoghby AO, Sheweita SA, et al. Self-assembled amphiphilic zein-lactoferrin micelles for tumor targeted co-delivery of rapamycin and wogonin to breast cancer. Eur J Pharm Biopharm. 2018;128:156-169.

20. Elzoghby AO, El-Lakany SA, Helmy MW, Abu-Serie MM, Elgindy NA. Shell-crosslinked zein nanocapsules for oral codelivery of exemestane and resveratrol in breast cancer therapy. Nanomedicine (Lond). 2017;12(24): 2785-2805.

21. Donnelly LE, Newton R, Kennedy GE, et al. Anti-inflammatory effects of resveratrol in lung epithelial cells: molecular mechanisms. $A m J$ Physiol Lung Cell Mol Physiol. 2004;287(4):L774-L783. 
22. Bishayee A. Cancer prevention and treatment with resveratrol: from rodent studies to clinical trials. Cancer Prev Res (Phila). 2009;2(5):409-418.

23. Varoni EM, Lo Faro AF, Sharifi-Rad J, Iriti M. Anticancer molecular mechanisms of resveratrol. Front Nutr. 2016;3:8.

24. Hamza RZ, El-Shenawy NS. Anti-inflammatory and antioxidant role of resveratrol on nicotine-induced lung changes in male rats. Toxicol Rep. 2017;4:399-407.

25. Elzoghby A. Editorial (Thematic issue: nanocarriers based on natural polymers as platforms for drug and gene delivery applications). Curr Pharm Des. 2016;22(22):3303-3304.

26. Elzoghby A, Freag M, Mamdouh H, Elkhodairy K. Zein-based nanocarriers as potential natural alternatives for drug and gene delivery: Focus on cancer therapy. Curr Pharm Des. 2017;23(35):5261-5271.

27. Chen RS, Ko JC, Chiu HC, et al. Pemetrexed downregulates ERCC1 expression and enhances cytotoxicity effected by resveratrol in human nonsmall cell lung cancer cells. Naunyn Schmiedebergs Arch Pharmacol. 2013;386(12):1047-1059.

28. Summerlin N, Soo E, Thakur S, Qu Z, Jambhrunkar S, Popat A. Resveratrol nanoformulations: challenges and opportunities. Int J Pharm. 2015;479(2):282-290.

29. Angelova A, Angelov B, Mutafchieva R, Lesieur S, Couvreur P. Self-assembled multicompartment liquid crystalline lipid carriers for protein, peptide, and nucleic acid drug delivery. Acc Chem Res. 2011 44(2):147-156.

30. Freag MS, Elnaggar YS, Abdelmonsif DA, Abdallah OY. Stealth, biocompatible monoolein-based lyotropic liquid crystalline nanoparticles for enhanced aloe-emodin delivery to breast cancer cells: in vitro and in vivo studies. Int J Nanomedicine. 2016;11(11):4799-4818.

31. Liu R, Wang S, Fang S, et al. Liquid crystalline nanoparticles as an ophthalmic delivery system for tetrandrine: development, characterization, and in vitro and in vivo evaluation. Nanoscale Res Lett. 2016;11(1):254.

32. Elzoghby AO,Abd-Elwakil MM, Abd-ElsalamK, ElsayedMT,Hashem Y, Mohamed O. Natural polymeric nanoparticles for brain-targeting: implications on drug and gene delivery. Curr Pharm Des. 2016;22(22): 3305-3323.

33. Elzoghby AO, Hemasa AL, Freag MS. Hybrid protein-inorganic nanoparticles: from tumor-targeted drug delivery to cancer imaging. $J$ Control Release. 2016;243:303-322.

34. Gaber M, Medhat W, Hany M, Saher N, Fang JY, Elzoghby A. Proteinlipid nanohybrids as emerging platforms for drug and gene delivery: challenges and outcomes. J Control Release. 2017;254:75-91.

35. Sabra S, Abdelmoneem M, Abdelwakil M, et al. Self-assembled nanocarriers based on amphiphilic natural polymers for anti-cancer drug delivery applications. Curr Pharm Des. 2017;23(35):5213-5229.

36. Spicer PT, Hayden KL, Lynch ML, Ofori-Boateng A, Burns JL. Novel process for producing cubic liquid crystalline nanoparticles (Cubosomes). Langmuir. 2001;17(19):5748-5756.

37. Devrim B, Bozkir A. Design and evaluation of hydrophobic ion-pairing complexation of lysozyme with sodium dodecyl sulfate for improved encapsulation of hydrophilic peptides/proteins by lipid-polymer hybrid nanoparticles. J Nanomed Nanotechnol. 2015;6(1):259.

38. Meyer JD, Manning MC. Hydrophobic ion pairing: altering the solubility properties of biomolecules. Pharm Res. 1998;15(2):188-193.

39. Swarnakar NK, Thanki K, Jain S. Bicontinuous cubic liquid crystalline nanoparticles for oral delivery of doxorubicin: implications on bioavailability, therapeutic efficacy, and cardiotoxicity. Pharm Res. 2014; 31(5):1219-1238.

40. Elzoghby AO, Mostafa SK, Helmy MW, ElDemellawy MA, Sheweita SA. Superiority of aromatase inhibitor and cyclooxygenase-2 inhibitor combined delivery: hyaluronate-targeted versus PEGylated protamine nanocapsules for breast cancer therapy. Int J Pharm. 2017;529(1-2): 178-192.

41. Swarnakar NK, Thanki K, Jain S. Bicontinuous cubic liquid crystalline nanoparticles for oral delivery of doxorubicin: implications on bioavailability, therapeutic efficacy, and cardiotoxicity. Pharm Res. 2014;31(5): 1219-1238.
42. Elgindy N, Elkhodairy K, Molokhia A, Elzoghby A. Biopolymeric nanoparticles for oral protein delivery: design and in vitro evaluation. J Nanomed Nanotechnol. 2011;02(03):110.

43. Elzoghby AO, Mostafa SK, Helmy MW, Eldemellawy MA, Sheweita SA. Multi-reservoir phospholipid shell encapsulating protamine nanocapsules for co-delivery of letrozole and celecoxib in breast cancer therapy. Pharm Res. 2017;34(9):1956-1969.

44. Khattab SN, Abdel Naim SE, El-Sayed M, et al. Design and synthesis of new s-triazine polymers and their application as nanoparticulate drug delivery systems. New J Chem. 2016;40(11):9565-9578.

45. Elzoghby AO, Vranic BZ, Samy WM, Elgindy NA. Swellable floating tablet based on spray-dried casein nanoparticles: near-infrared spectral characterization and floating matrix evaluation. Int J Pharm. 2015;491(1-2):113-122.

46. Freag MS. Hyaluronate-lipid nanohybrids: fruitful harmony in cancer targeting. Curr Pharm Des. 2017;23(35):5283-5291.

47. Freag MS, Elnaggar YS, Abdelmonsif DA, Abdallah OY. Layer-bylayer-coated lyotropic liquid crystalline nanoparticles for active tumor targeting of rapamycin. Nanomedicine (Lond). 2016;11(22):2975-2996.

48. Deshpande S, Venugopal E, Ramagiri S, Bellare JR, Kumaraswamy G, Singh N. Enhancing cubosome functionality by coating with a single layer of poly-e-lysine. ACS Appl Mater Interfaces. 2014;6(19): $17126-17133$.

49. Jain V, Swarnakar NK, Mishra PR, et al. Paclitaxel loaded PEGylated gleceryl monooleate based nanoparticulate carriers in chemotherapy. Biomaterials. 2012;33(29):7206-7220.

50. Deshpande S, Singh N. Influence of cubosome surface architecture on its cellular uptake mechanism. Langmuir. 2017;33(14):3509-3516.

51. Harush-Frenkel O, Debotton N, Benita S, Altschuler Y. Targeting of nanoparticles to the clathrin-mediated endocytic pathway. Biochem Biophys Res Commun. 2007;353(1):26-32.

52. Li Q, Yano S, Ogino H, et al. The therapeutic efficacy of anti vascular endothelial growth factor antibody, bevacizumab, and pemetrexed against orthotopically implanted human pleural mesothelioma cells in severe combined immunodeficient mice. Clin Cancer Res. 2007;13(19): 5918-5925.

53. Sahin E, Baycu C, Koparal AT, Burukoglu Donmez D, Bektur E. Resveratrol reduces IL-6 and VEGF secretion from co-cultured A549 lung cancer cells and adipose-derived mesenchymal stem cells. Tumour Biol. 2016;37(6):7573-7582.

54. Luo T, Loira-Pastoriza C, Patil HP, et al. PEGylation of paclitaxel largely improves its safety and anti-tumor efficacy following pulmonary delivery in a mouse model of lung carcinoma. J Control Release. 2016; 239:62-71.

55. Wu J, Deng C, Meng F, Zhang J, Sun H, Zhong Z. Hyaluronic acid coated PLGA nanoparticulate docetaxel effectively targets and suppresses orthotopic human lung cancer. J Control Release. 2017;259: 76-82.

56. Kitada M, Koya D. Renal protective effects of resveratrol. Oxid Med Cell Longev. 2013;2013(1):1-7.

57. Wang Y, Jiang Y, Fan X, et al. Hepato-protective effect of resveratrol against acetaminophen-induced liver injury is associated with inhibition of CYP-mediated bioactivation and regulation of SIRT1-p53 signaling pathways. Toxicol Lett. 2015;236(2):82-89.

58. Chen WM, Shaw LH, Chang PJ, et al. Hepatoprotective effect of resveratrol against ethanol-induced oxidative stress through induction of superoxide dismutase in vivo and in vitro. Exp Ther Med. 2016;11(4): 1231-1238.

59. Osman AM, Telity SA, Damanhouri ZA, et al. Chemosensitizing and nephroprotective effect of resveratrol in cisplatin-treated animals. Cancer Cell Int. 2015;15(1):6. 


\section{Supplementary materials \\ Methodology}

HPLC method for the assay of pemetrexed (PMX) and resveratrol (RSV)

A novel, accurate, precise, and sensitive HPLC method was developed and validated for simultaneous determination of both RSV and PMX using Agilent 1260 Infinity HPLC system equipped with a quaternary pump, an autosampler, vacuum degasser, diode array detector, and Agilent Chemstation data-processing system. The HPLC analysis was carried out with an Inertsil ${ }^{\circledR}$ ODS-3 reversed-phase column $(250 \times 4.6 \mathrm{~mm}, 5 \mu \mathrm{m}$; GL Sciences Inc, Torrance, CA, USA). The column was maintained at room temperature. For chromatographic elution, the injection volume was 20 $\mu \mathrm{L}$. A step gradient was utilized for elution in which the mobile phase consisted of acetonitrile: dibasic phosphate buffer (pH adjusted to 4.9 using orthophosphoric acid) with the following ratio 15:85 to 6 minutes then converted into 50:50 gradually over 0.5 minutes, and the flow rate was 1.5 $\mathrm{mL} / \mathrm{min}$. Total run time was 10 minutes; PMX was eluted at 4.8 minutes and RSV at 9.8 minutes. PMX was detected at $225 \mathrm{~nm}$, while RSV was detected at $306 \mathrm{~nm}$.

\section{Evaluation of in vitro cytotoxicity}

In vitro cytotoxicity of free PMX, free RSV, free combined PMX/RSV solution, blank liquid crystalline nanoparticles (LCNPs), and ion-paired PMX-RSV-LCNPs was evaluated in A549 lung cancer cells using MTT assay. ${ }^{1}$ Briefly, 1,000-5,000 cells/well were seeded in 96-well culture plates containing $100 \mu \mathrm{L}$ of cell line-specific medium and incubated at $37^{\circ} \mathrm{C}$ in a $5 \% \mathrm{CO}_{2}$ atmosphere. The cells were then treated with each formulation at concentrations ranged from 0.468 to $30 \mu \mathrm{g} / \mathrm{mL}$ of each PMX and/or RSV and incubated at $37^{\circ} \mathrm{C}$ for 24 and 48 hours. Then, cells were subjected to the MTT analysis for cell viability determination. The optic density was measured at $490 \mathrm{~nm}$ on a microplate reader with control wells containing only cell culture medium. The viability data were analyzed in GraphPad Prism, and the $\mathrm{IC}_{50}$ value for each system was calculated. Each $\mathrm{IC}_{50}$ value was calculated from $n=6$ viability measurements per concentration. $\mathrm{IC}_{50}$ was calculated after 24 and 48 hours.

\section{In vitro cellular uptake}

A549 lung cells were seeded on CELL view ${ }^{\mathrm{TM}}$ slide at a density of 3,000 cells/well and left overnight to allow enough time for cell attachment. Cells were incubated with fresh medium containing free coumarin-6 dye and coumarin-6-loaded LCNPs at the concentration of $0.08 \% \mathrm{w} / \mathrm{w}$ coumarin- 6 (with respect to lipid). After incubation for 4 and 24 hours, the medium was removed and cells were rinsed twice using PBS to eliminate any extracellular residues. Then, cells were fixed using $4 \%$ paraformaldehyde solution in dark. The nuclei were stained using 2-(4-ethoxyphenyl)-6-[6-(4-methylpiperazin1-yl)-1H-benzimidazol-2-yl]-1H-benzimidazole (Hoechst AG, Frankfurt, Germany) containing DPX (DePeX [Distrene 80: A commercial polystyrene, a plasticizer, e.g., dibutyl phthalate and xylene]) as mounting medium. Finally, the intracellular uptake of optimized LCNPs into A549 cell line was visualized via confocal laser scanning microscopy (DMi8; Leica Microsystems, Wetzlar, Germany) using the following filter set: excitation wavelength, $450 \mathrm{~nm}$; emission wavelength, $505 \mathrm{~nm}$. All obtained confocal images were analyzed using Leica AF software. Moreover, the internalization of particles within each section of the cells at various cell depths was visualized using three-dimensional cellular images per time (z-stacks), where serial sections were taken across 30 fields and stacked with cross-sectional slices $(25 \mu \mathrm{m})$ perpendicular to the plane of the cell monolayer midpoint (z-axis). ${ }^{2,3}$

\section{Results and discussions}

\section{HPLC method for the assay of PMX and RSV}

Several attempts were carried out for simultaneous quantitation of PMX and RSV in mixture. Isocratic elution using different proportions of mobile phases incluwding methanol, acetonitrile, acidified water, and buffer at different ratios was not enough to provide satisfactory separation for the mixture's components. Therefore, gradient elution was applied to separate peaks of both drugs. ${ }^{4}$ In the literature, various methods were reported for individual quantitation of PMX and RSV in formulations and biological fluids. ${ }^{4-8}$ The optimized mobile phase composed of HPLC grade acetonitrile: dibasic phosphate buffer ( $\mathrm{pH}$ adjusted to 4.9 using orthophosphoric acid) with the following ratio 15:85 to 6 minutes and then converted into 50:50 gradually over 0.5 minutes; flow rate was $1.5 \mathrm{~mL} / \mathrm{min}$. A rapid, sensitive, simple, and efficient method was developed for simultaneous determination of RSV and PMX in stock solution and nanoformulations. The principal peak obtained with the standard solution was at $225 \mathrm{~nm}$ which was selected as the $\lambda_{\text {max }}$ of the PMX, whereas the major peak of RSV was detected at $\lambda_{\text {max }}$ $306 \mathrm{~nm}$. A good linearity was shown by the calibration curve obtained from 0.4 to $2 \mathrm{mg} \%$ PMX and RSV concentration (Figure S1). The coefficient of determination (R2) of 0.9997 was obtained from the linear regression analysis of the data (peak area vs concentration). Selected chromatograms were displayed (Figure S2), in which retention time of PMX was 


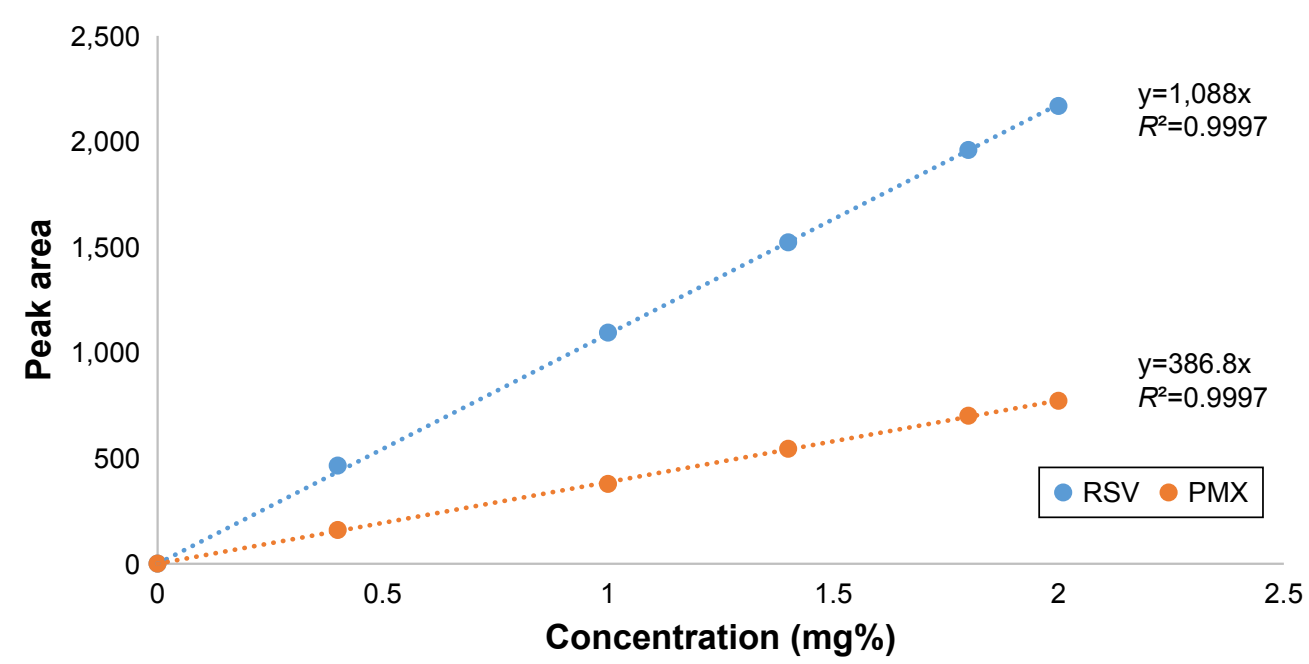

Figure SI HPLC calibration curve of PMX and RSV in methanol at $\lambda_{\text {max }} 225$ and $306 \mathrm{~nm}$, respectively. Abbreviations: PMX, pemetrexed; RSV, resveratrol.

4.427 minutes and of RSV was 11.87 minutes so that the total run time was 12 minutes.

As summarized in Table S1, the HPLC method was validated according to International Conference on Harmonization (IHC) guidelines for intra- and inter-day precision and accuracy. The low values of relative standard deviation
(RSD) as well as \% error of mean $<2$ confirmed the precision and accuracy of the developed HPLC method. Limit of detection was $0.2 \mathrm{mg} \%$, while the limit of quantitation was 0.4 $\mathrm{mg} \%$. The proposed assays were also successfully applied to evaluate the entrapment efficiency (EE) of the novel LCNPs for the co-delivery of PMX and RSV for lung cancer.

A

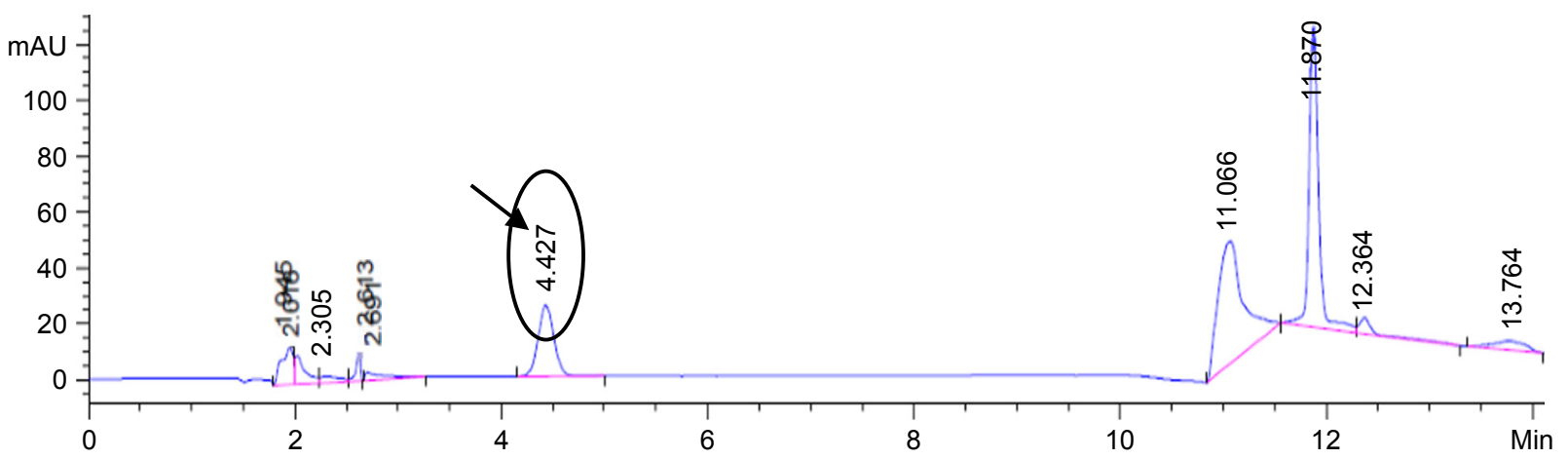

B

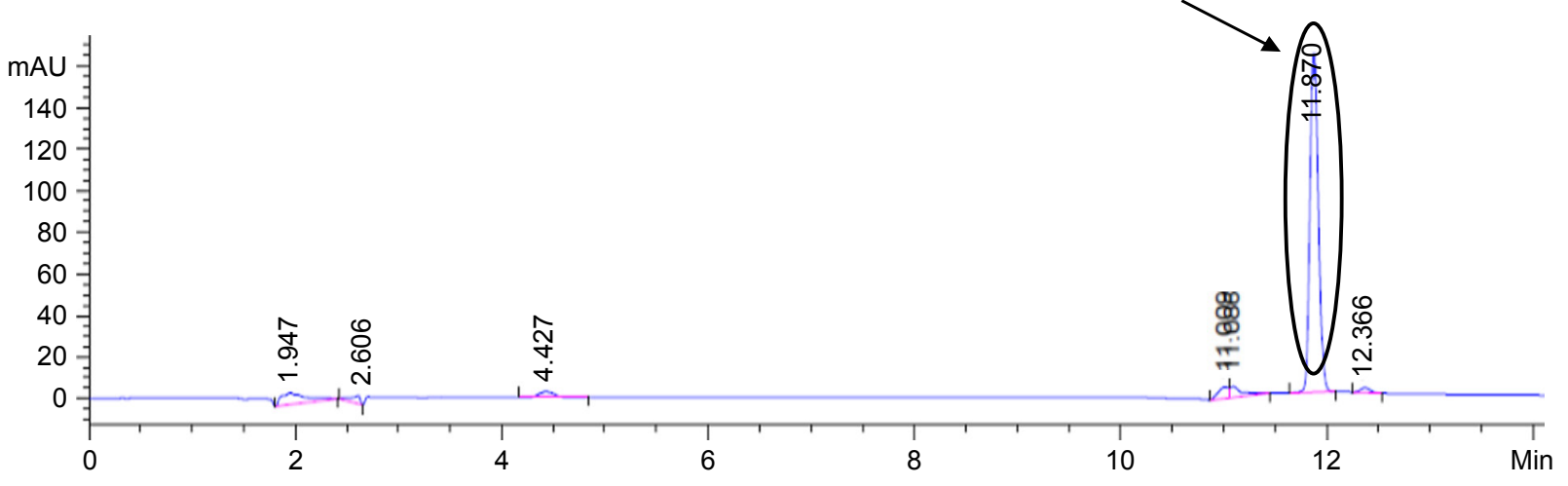

Figure S2 Selected chromatogram of (A) PMX and (B) RSV displaying retention time their corresponding $\lambda_{\max }$. Abbreviations: PMX, pemetrexed; RSV, resveratrol. 


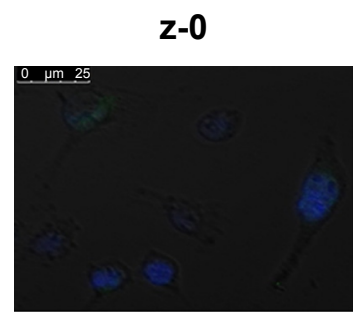

z-12

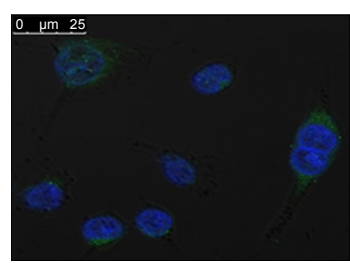

z-24

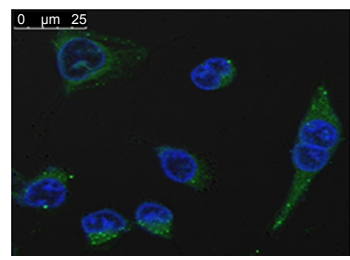

$z-3$

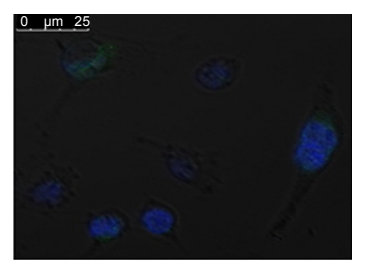

z-15

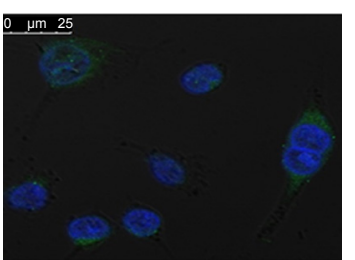

Z-27

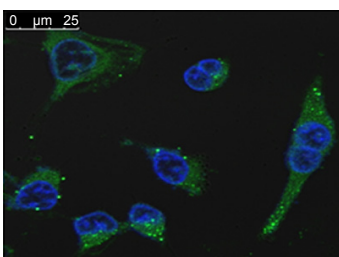

z-6

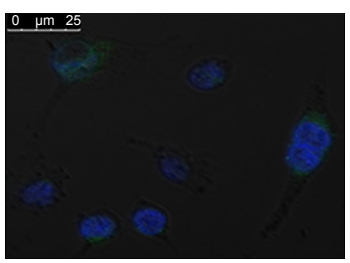

z-18

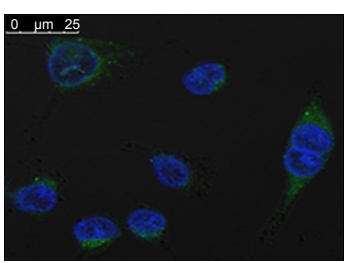

z-28

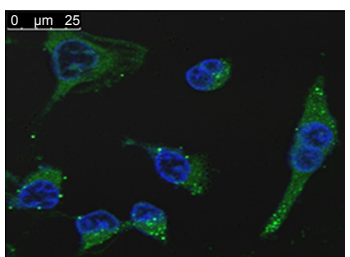

$z-9$

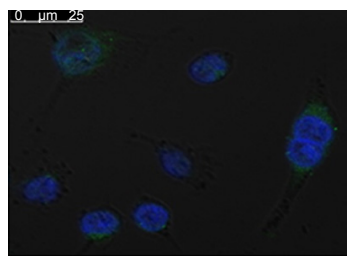

z-21

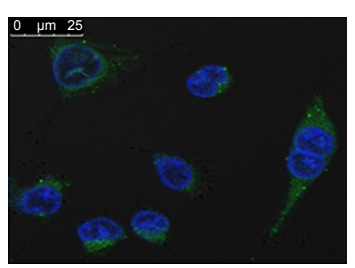

z-29

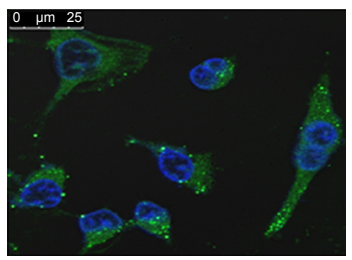

Figure S3 Live uptake of ion-paired LCNPs in A549 cells in which three-dimensional time laps images distinguishing between cystolic and perinuclear accumulation of I 78 nm LCNPs of 30 z-stacks inside the cells in the z-direction along the total depth measured (z-stack $=30$ slices at $25 \mu \mathrm{m}$ per slices).

Note: The individual stacks were assembled as video displaying the oscillation of green fluorescence along the cross-sectional internalization of LCNPs from the apical to basolateral cellular membrane represented from z-0 to z-29.

Abbreviation: LCNPs, liquid crystalline nanoparticles.

Table SI Intra- and inter-day precision and accuracy of PMX and RSV ( $\mathrm{n}=5$ for each concentration)

\begin{tabular}{|c|c|c|c|c|c|c|c|}
\hline \multirow{2}{*}{$\begin{array}{l}\text { Concentration } \\
(\mathrm{mg} \%)\end{array}$} & \multirow[t]{2}{*}{ Drug } & \multicolumn{3}{|c|}{ Intra-day $(n=5)$} & \multicolumn{3}{|c|}{ Inter-day $(n=5)$} \\
\hline & & Mean \pm SD & RSD & $\%$ error & Mean \pm SD & RSD & \% error \\
\hline \multirow[t]{2}{*}{0.6} & PMX & $0.611 \pm 0.001$ & 0.262 & 1.940 & $0.604 \pm 0.011$ & 1.903 & 0.733 \\
\hline & RSV & $0.61 \mathrm{I} \pm 0.01 \mathrm{I}$ & 1.864 & 1.916 & $0.588 \pm 0.003$ & 0.619 & -1.933 \\
\hline \multirow[t]{2}{*}{0.8} & PMX & $0.80 \mathrm{I} \pm 0.00 \mathrm{I}$ & 0.127 & 0.140 & $0.805 \pm 0.012$ & 1.600 & 0.625 \\
\hline & RSV & $0.787 \pm 0.016$ & 2.084 & -1.507 & $0.790 \pm 0.012$ & 1.553 & -1.201 \\
\hline \multirow[t]{2}{*}{ I } & PMX & $0.999 \pm 0.001$ & 0.154 & -0.043 & $0.998 \pm 0.004$ & 0.459 & -0.201 \\
\hline & RSV & $0.996 \pm 0.018$ & 1.823 & -0.401 & $0.990 \pm 0.015$ & 1.613 & -0.980 \\
\hline \multirow[t]{2}{*}{1.8} & PMX & I.797士0.00I & 0.080 & -0.133 & $1.814 \pm 0.017$ & 0.989 & 0.777 \\
\hline & RSV & $1.806 \pm 0.020$ & 1.107 & 0.350 & $1.799 \pm 0.017$ & 0.957 & -0.055 \\
\hline \multirow[t]{2}{*}{2} & PMX & $1.999 \pm 0.001$ & 0.052 & -0.007 & $1.974 \pm 0.035$ & 1.776 & -1.301 \\
\hline & RSV & $1.994 \pm 0.019$ & 0.994 & -0.270 & $1.999 \pm 0.009$ & 0.463 & -0.050 \\
\hline
\end{tabular}

Abbreviations: PMX, pemetrexed; RSV, resveratrol.

\section{References}

1. Khattab SN, Naim SEA, El-Sayed M, et al. Design and synthesis of new s-triazine polymers and their application as nanoparticulate drug delivery systems. New J Chem. 2016;40(11):9565-9578.

2. Yadav KS, Jacob S, Sachdeva G, Sawant KK. Intracellular delivery of etoposide loaded biodegradable nanoparticles: cytotoxicity and cellular uptake studies. J Nanosci Nanotechnol. 2011;11(8):6657-6667.

3. Teskač K, Kristl J. The evidence for solid lipid nanoparticles mediated cell uptake of resveratrol. Int J Pharm. 2010;390(1):61-69.

4. El-Lakany SA, Elzoghby AO, Elgindy NA, Hamdy DA. HPLC methods for quantitation of exemestane-luteolin and exemestane-resveratrol mixtures in nanoformulations. J Chromatogr Sci. 2016;54(8):1282-1289.
5. Rivory LP, Clarke SJ, Boyer M, Bishop JF. Highly sensitive analysis of the antifolate pemetrexed sodium, a new cancer agent, in human plasma and urine by high-performance liquid chromatography. JChromatogr $B$ Biomed Sci Appl. 2001;765(2):135-140.

6. Singh G, Pai RS, Pandit V. Development and validation of a HPLC method for the determination of trans-resveratrol in spiked human plasma. J Adv Pharm Technol Res. 2012;3(2):130-135.

7. Warner A, Piraner I, Weimer H, White K. Development of a purity control strategy for pemetrexed disodium and validation of associated analytical methodology. J Pharm Biomed Anal. 2015;105:46-54.

8. Katsagonis A, Atta-Politou J, Koupparis MA. HPLC method with UV detection for the determination of trans-resveratrol in plasma. J Liquid Chromatogr Relat Technol. 2005;28(9):1393-1405. 
International Journal of Nanomedicine

Dovepress

\section{Publish your work in this journal}

The International Journal of Nanomedicine is an international, peerreviewed journal focusing on the application of nanotechnology in diagnostics, therapeutics, and drug delivery systems throughou the biomedical field. This journal is indexed on PubMed Central, MedLine, CAS, SciSearch ${ }^{\circledR}$, Current Contents ${ }^{\circledR} /$ Clinical Medicine,
Journal Citation Reports/Science Edition, EMBase, Scopus and the Elsevier Bibliographic databases. The manuscript management system is completely online and includes a very quick and fair peer-review system, which is all easy to use. Visit http://www.dovepress.com/ testimonials.php to read real quotes from published authors.

Submit your manuscript here: http://www.dovepress.com/international-journal-of-nanomedicine-journal 